\title{
Coherent mapping of position and head direction across auditory and visual cortex
}

Paul EC Mertens ${ }^{1}$, Pietro Marchesi ${ }^{1}$, Matthijs Oude Lohuis ${ }^{1}$, Quincy Krijger ${ }^{1}$, Cyriel MA Pennartz ${ }^{1,2}$ and Carien S Lansink ${ }^{1,2}$

\author{
${ }^{1}$ Swammerdam Institute for Life Sciences, Center for Neuroscience, Faculty of Science, University of \\ Amsterdam, Science Park 904, 1098 XH, Amsterdam, the Netherlands. \\ ${ }^{2}$ Research Priority Program Brain and Cognition, University of Amsterdam, Science Park 904, 1098 XH, \\ Amsterdam, the Netherlands.
}

Corresponding Author: Cyriel MA Pennartz, c.m.a.pennartz@uva.nl

\begin{abstract} Neurons in primary visual cortex (V1) may not only signal current visual input but also relevant contextual 4 information such as reward expectancy and the subject's spatial position. Such location-specific representations need not be restricted to V1 but could participate in a coherent mapping throughout sensory cortices. Here we show that spiking activity in primary auditory cortex (A1) and lateral, secondary visual cortex (V2L) of freely moving rats coherently represents a location-specific mapping in a sensory

8 detection task performed on a figure-8 maze. Single-unit activity of both areas showed extensive 9 similarities in terms of spatial distribution, reliability and position coding. Importantly, reconstructions of 10 subject position on the basis of spiking activity displayed decoding errors that were correlated between 11 areas in magnitude and direction. In addition to position, we found that head direction, but not locomotor 12 speed or head angular velocity, was an important determinant of activity in A1 and V2L. Finally, pairs of 13 units within and across areas showed significant correlations in instantaneous variability of firing rates 14 (noise correlations). These were dependent on the spatial tuning of cells as well as the spatial position of 15 the animal. We conclude that sensory cortices participate in coherent, multimodal representations of the 16 subject's sensory-specific location. These may provide a common reference frame for distributed cortical 17 sensory and motor processes and may support crossmodal predictive processing.




\section{Introduction}

Early sensory cortical areas were long viewed to primarily function as collections of unisensory feature detectors (DiCarlo and Cox, 2007; Felleman and Van Essen, 1991; Hubel and Wiesel, 1962; Miller, 2016). More recently, single unit recordings in awake, behaving animals have shown responses in primary auditory (A1) and visual cortex (V1) to a wide variety of perceptual and behavioral factors, suggesting these areas have functions beyond unimodal sensory processing. In rodent $\mathrm{V} 1$, these include responses to reward and reward timing (Shuler and Bear, 2006), reward predictive stimuli (Goltstein et al., 2013), running speed (Ayaz et al., 2013; Niell and Stryker, 2010), head orienting movements (Guitchounts et al., 2020) and responses which are causal to visually cued action timing (Namboodiri et al., 2015). Additionally, a growing number of studies show that many V1 neurons display location-selective spiking activity (Ji and Wilson, 2007), coding the animal's position along real (Haggerty and Ji, 2015) and virtual linear tracks (Fiser et al., 2016; Fournier et al., 2020; Pakan et al., 2018; Saleem et al., 2018). Various studies report spatial and temporal correlations in activity of V1 and hippocampal CA1, including correlated errors in position decoding (Fournier et al., 2020; Saleem et al., 2018), correlated trial-by-trial shifts in preferred spiking locations (Haggerty and Ji, 2015) and significant spike-phase coherence of V1 spiking and hippocampal theta oscillations (Fournier et al., 2020).

A similarly broad variety of single unit correlates is observed in A1, including activity selective for visual task-cues (Brosch et al., 2005), behavioral demands (Scheich et al., 2007), stimulus expectation (Jaramillo and Zador, 2011), reward (Scheich et al., 2007) and instrumental action (Niwa et al., 2012). Much remains currently unknown about the functional role and origins of such 'extra-modal' activity correlates, including whether they primarily contribute to local sensory processes or reflect crossmodal interactions in service of more general and modality-independent cortical functions. While spiking correlates to stimulus location are present in auditory cortex (Town et al., 2017), no activity selective for the spatial position of the subject has hitherto been reported for A1. This would be expected if the underlying mechanisms reflect general functions of cortical sensory processing, including the maintenance and updating of a coherent representation of space or of current and future sensory states across sensory domains (Fiser et al., 2016; Friston, 2005; Gavornik and Bear, 2014; Pennartz et al., 2019; Rao and Ballard, 1999).

To determine whether location-selective spiking activity exists outside of the visual cortical system and whether such activity provides a coherent representation across sensory modalities, we analyzed single-unit data recorded simultaneously from two anatomically connected, sensory cortical areas of freely moving rats: primary auditory cortex (A1) and lateral, secondary visual cortex (V2L). We 
51 show spatially localized firing patterns in large proportions of $\mathrm{V} 2 \mathrm{~L}$ and $\mathrm{A} 1$ single units that are reliable over

52 time. Firing patterns in each area collectively tiled the entire behavioral track, so that every location was

53 marked by activity of a subset of neurons. Reconstructions of the rat's position afforded by the spiking

54 activity of each area showed reconstruction errors that were correlated in magnitude and direction,

55 thereby indicating that representations in A1 and V2L are coherent. Cross-areal coordination of location-

56 specific representations was further indicated by strong noise-correlations in spiking activity of the vast

57 majority of single-unit pairs which showed a clear pattern of dependence on both the spatial tuning of the

58 units as well as animal position. Our freely moving paradigm allowed us to establish the contributions of

59 position, head direction and their temporal derivatives to location-selective spiking activity in early

60 sensory cortices dedicated to different modalities. Our results uncover striking similarities as well as

61 quantitative differences in location-selective neural activity of $A 1$ and V2L, suggesting that such activity

62 supports common functions in coordinated mapping of sensory and contextual representations across

63 different sensory modalities.

64 


\section{Results}

66 We investigated the responsiveness of neurons in lateral, secondary visual cortex (V2L) and primary 67 auditory cortex (A1) to spatial location when rats were running on a rectangular, figure-8 shaped track 68 (Fig.1A). On the track, rats performed an audio-visual discrimination task in which they earned reward by 69 responding to the most salient stimulus out of two by running from the stimulus presentation site to the reward well on the track side corresponding to the location of that stimulus (Fig. 1 A-B). Our analyses were primarily based on the spatial components of the rats' behavior, regardless of task performance. We refer to "location-selective activity" or a "location correlate" if the neuron's spiking activity was reliably modulated by the rat's body location over the course of a recording session. We emphasize that this definition includes not only responses to allocentric position, but also to specific conjunctions of locally available sensory cues and task-related information.

We made simultaneous recordings from both $\mathrm{V} 2 \mathrm{~L}$ and $\mathrm{A} 1$, with a total of 526 single units from V2L and 603 units from A1 across 17 recording sessions from 3 rats. Units in both A1 and V2L showed all further analyses, we selected the units with sufficient firing on the track, i.e. peak firing rate above 2 $\mathrm{Hz}$ in the spatial map, which amounted to 400 units from V2L and 413 from A1. The locations of the tetrode endpoints were verified with histology (Supplementary Fig. 1). The endpoints of 20 tetrodes were located in A1, while an additional two endpoints targeted at A1 were located in the adjacent dorsal secondary auditory cortex. Endpoints for 19 tetrodes targeted at V2L were located in that area, while one was

84 located in the adjacent dorsal posterior parietal cortex.

The majority of $A 1$ and V2L single units displays localized spiking activity

87 First, we assessed whether neurons in A1 and V2L displayed spatially localized firing patterns. Visual inspection of rate maps of the spatial firing distribution of individual neurons indicated that some units in A1 and V2L showed increased spiking activity in a single, concentrated location on the track, whereas other units displayed modulations of spiking activity at multiple areas or across a larger area of the track (Fig. 2A-B top panels). An important constraint for a unit to reliably code location is that the firing activity

92 at that location is consistently, rather than incidentally, present across individual traversals through that 93 location. Therefore, we assessed the reliability of each unit by computing pairwise correlations between 94 all single-trial rate maps of that unit and comparing the observed mean pairwise correlation with a 95 shuffled distribution. A unit was considered spatially stable if its observed mean correlation was larger 96 than $95 \%$ of the shuffled distribution. The proportions of spatially stable units were very similar for A1 
and $\mathrm{V} 2 \mathrm{~L}$ and comprised on average about $70 \%$ of the total population (Fig. $2 \mathrm{C} ; \mathrm{A} 1: 0.69$ (Cl: $0.49,0.84$ ), V2L: $0.72(\mathrm{Cl}: 0.31,0.94), F(1,32)=0.072, p=0.79$, ANOVA). The degree of spatial stability of a unit can be quantified using a spatial stability index (SSI), defined as the number of standard deviations which the observed mean correlation was removed from the shuffled distribution (Fig. 2D). The SSI of spatially stable units was similar between A1 and V2L (Fig. 2D; A1: 12.7 a.u. (CI: 8.39, 15.44), V2L: 14.3 a.u. (CI: 10.4, 24.4), $F(1,1194)=0.44, p=0.50$, ANOVA). In summary, large fractions of A1 and V2L units display spatially stable activity patterns with high reliability.

Individual firing fields were then identified for spatially stable units as localized increases in mean spiking activity of a unit on the linearized representation of the track (Fig. 1A, Fig. 2A-B). Whereas neurons in $\mathrm{A} 1$ and $\mathrm{V} 2 \mathrm{~L}$ exhibited a similar number of around 3 firing fields per unit (Fig. 2E; $\mathrm{A} 1: 3.17$ (CI: 2.97, 3.39), V2L: $3.40(\mathrm{Cl}: 3.20,3.61), F(1,588)=2.22, p=0.14$, ANOVA), the average length of firing fields was significantly smaller in V2L than in A1 (Fig. 2F; A1: $50.5 \mathrm{~cm}$ (Cl: 47.2, 54.1), V2L; $46.3 \mathrm{~cm}$ (Cl: 43.5, 49.4), $F(1,1939)=9.0, p=0.003, A N O V A)$, indicating that the spatial granularity of localized spiking activity is modestly finer in V2L than A1.

The extent to which units show spatially localized firing can be expressed as the information about the rat's position that is conveyed by a single spike, which is quantified as spatial information (Skaggs et al., 1992). In line with the smaller firing fields in V2L, the spatial information was significantly higher for V2L units (Fig. 2G; A1: 0.15 bits/spike (Cl: 0.12, 0.17), V2L: 0.24 bits/spike $(\mathrm{Cl}: 0.20,0.28), F(1,556)=16.5$, $p=5 * 10^{-5}$, ANOVA).

\section{Both $A 1$ and V2L neurons respond to discrete auditory and visual stimuli}

118 Besides showing location-selective firing, subsets of units in both A1 and V2L responded with significant 119 firing-rate changes to the auditory and visual stimuli, presented as individual (unisensory) task cues (Fig. $1202 \mathrm{H}$; stimulus conditions were pooled for each modality; see Methods). As expected, a larger proportion of $A 1$ neurons responded to auditory than to visual stimuli in all rats ( $A 1$ auditory: 0.20 ( $\mathrm{Cl}: 0.15,0.28), A 1$ visual: $0.07(\mathrm{Cl}: 0.04,0.13), F(1,30)=10.34, p=0.003$, ANOVA). Additionally, responsiveness to visual

123 stimuli was more common in V2L than $\mathrm{A} 1$ (V2L visual: 0.15 (Cl: 0.09, 0.24), $F(1,30)=4.60, p=0.0402$,

124 ANOVA). Surprisingly, however, a comparable proportion of V2L neurons responded to both visual and 125 auditory (stimuli. (V2L auditory: $0.20,(\mathrm{Cl}: 0.15,0.26), \mathrm{F}(1,30)=0.93, \mathrm{p}=0.34$, ANOVA) and auditory responses were equally common in both areas $(F(1,30)=0.013, p=0.91$, ANOVA). Although A1 and V2L

127 responsiveness to discrete stimuli is not the focus of our current analyses, these results not only indicate 128 that responses to stimuli in more than one modality are common in both A1 and especially V2L, but also 
underscore the existence of substantial heterogeneity in cortical sensory selectivity. Finally, it should be noted that stable, localized activity was much more abundant in both areas than responses to sensory stimuli (A1: $p<10^{-15} ; \mathrm{V} 2 \mathrm{~L}: \mathrm{p}<10^{-13}$, binomial tests on pooled data).

134 The reliable coding of specific locations by individual units is necessary, but not sufficient for building a 135 representation of an environment or the sequence of sensory states an animal experiences when navigating across the track. Another prerequisite for either type of representation would be that the spatial distribution of firing fields in each area covers the entire series of locations traversed by the animal. Figure $3 \mathrm{~A}$ and $\mathrm{B}$ show the linear rate maps for all spatially stable units ordered by peak location for $\mathrm{A} 1$ and V2L, and reveal that firing-field peaks occur at every location along the track. We further analyzed the density of firing fields tiling all locations by computing the proportion of firing fields which include a specific spatial bin on the linearized track. The distributions of firing field densities confirm that the entire track was covered by $\mathrm{A} 1$ and V2L firing fields (Fig. 3A-B, bottom).

The joint rate maps and field density plots indicate similar firing-field distributions in $\mathrm{A} 1$ and V2L. Indeed, the field densities across spatial bins of the track were highly correlated between the areas (Fig. 3C; regression slope: $0.83(\mathrm{Cl}: 0.57,1.09), F(1,39.5)=3.2 p=0.007$, ANOVA). This strong correlation, however, may also be due to similar responses of $\mathrm{A} 1$ and $\mathrm{V} 2 \mathrm{~L}$ neurons to behavioral covariates such as locomotion speed, acceleration and head direction $\left(\theta_{\text {head }}\right)$. To correct for the potential influence of these covariates on field densities, we performed linear regression of spiking activity on running speed, acceleration, head direction and the angular change in head direction ( $\left.\Delta \theta_{\text {head }}\right)$ and repeated the detection of firing fields and the field density correlations on the residuals. Likewise, when using the model's residuals, field densities between $\mathrm{A} 1$ and V2L were highly correlated (regression slope: 0.71 (Cl:0.36, 1.06), $F(1,3.0)=16.1, p=0.028$, ANOVA). It is therefore unlikely that locomotion and head direction can fully

154 These analyses, however, did not take into account possible nonlinear relationships between location and behavioral covariates.

Temporal firing rate fluctuations are correlated between $A 1$ and V2L

158 Neurons with similar functional properties, such as location-selective tuning in hippocampal and V1 neurons, were shown to exhibit significant pairwise correlations of the instantaneous variability in spiking activity, often referred to as 'noise correlations' (Haggerty and Ji, 2015). As a first indication of a 
correlative interaction between spatially stable neurons within and across A1 and V2L, we tested how closely the precise firing rate between two units of a pair varied each time the rat ran through the associated firing fields. For each pair of units (A1-A1, V2L-V2L, A1-V2L) the Pearson correlation was computed between their instantaneous variability in spiking activity after subtraction of the mean activity in the associated spatial bin (i.e. noise correlation), as well as the Pearson correlation between the rate maps. Noise correlations were significantly different from 0 for very large proportions of neuronal pairs of each composition (fraction of total: A1-A1: 0.98, V2L-V2L: 0.98, A1-V2L: 0.97; permutation test, p<0.05). Almost half of the significantly correlated pairs showed negative noise correlations (A1-A1: 0.46, V2L-V2L: 0.47, A1-V2L: 0.45). Proceeding only with pairs showing significant noise correlations, we tested whether rate map similarity predicted noise correlations using a linear mixed model and correcting for sampling bias. Rate-map correlations had a significant, positive effect on noise correlations for all pair types (Fig. 3D-E; regression slopes and 95\% confidence bounds for A1-A1: $0.072(0.060,0.085), F(1,4.8)=123.9, p$ $=3.9 * 10^{-4}$, V2L-V2L: $0.066(0.051,0.082), F(1,4.17)=110.4, \mathrm{p}=7.4 * 10^{-4}, \mathrm{~A} 1-\mathrm{V} 2 \mathrm{~L}: 0.033(0.018,0.048)$, $F(1,3.67)=29.3, p=0.007$, ANOVA). The effect of rate map similarity was smaller for A1-V2L pairs than for the other pair types (A1-V2L slope vs. A1-A1 slope: $F(1,9386)=95.9, p<10^{-15}, A 1-V 2 L$ slope vs. V2LV2L slope: $F(1,10174)=83.2, p<10^{-15}$, ANOVA), which is in line with previous observations on noise correlation strength decreasing with anatomical distance observed in visual cortex of anesthetized cats, mice and macaques (Goltstein et al., 2015; Rosenbaum et al., 2017; Schulz et al., 2015; Smith and Kohn, 2008). Nonetheless, the V2L-A1 noise correlates and their predictability from rate-map correlations

Noise correlations within and between areas show location-dependent structure

183 Correlated firing rate fluctuations can affect the coding of information in populations of neurons (Ecker et 184 al., 2010; Hansen et al., 2012; Montijn et al., 2016; Zohary et al., 1994). The strength of correlated variability in the visual system was found to depend on both the tuning of neurons and the presented stimuli, with similarly tuned neurons displaying stronger correlated variability specifically for their preferred stimuli and reduced correlations for orthogonal stimuli, thereby ameliorating the potentially detrimental effect of correlated variability on population coding (Averbeck et al., 2006; Franke et al., 2016; Lin et al., 2015; Montijn et al., 2016). If the function of location-selective activity in A1 and V2L is to transmit information related to the animal's position to downstream areas, correlated variability between

192 for each significantly correlated pair we divided all spatial bins of the track into three categories: bins that 
193

occur inside firing fields of both units of the pair ('shared field'), bins that exclusively occur in a firing field of one of the two units ('one-field'), and bins that are outside of firing fields of both units ('out of field'). The effect of firing-field overlap on noise correlations for each area was examined using a linear mixed model. Separate models were constructed for positive and negative noise correlations, since the mean noise correlations of individual groups tended to 0 , while a model based on absolute noise correlations provided a poorer fit to the data.

The magnitude of noise correlations followed a consistent pattern across cell pair types with respect to the type of bin, with shared field bins showing stronger correlations than one-field bins (figure $3 F$, positive correlations, shared field bins mean $=0.061$, one-field bins mean $=0.046, F(1,3.2)=139.5, p$ $=4.7 * 10^{-4}$, negative correlations, shared field bins mean $=-0.049$, one-field bins mean $=-0.035, F(1,8.9)$ $=233.9, p=3.5 * 10^{-8}$, ANOVA). Additionally, shared field bins showed stronger correlations than out of field bins (figure 3F, positive correlations: out of field bins mean $=0.052, F(1,2.9)=43.3, p=0.008$, negative correlations: out of field bins mean $=-0.040, F(1,2.7)=41.9, p=0.005$, ANOVA). Finally, out of field bins showed stronger correlations than one-field bins (figure $3 F$, positive correlations: $F(1,29.3)=$ 28.7, $p=3.0 * 10^{-6}$, negative correlations: $F(1,2.7)=14.8, p=0.04$, ANOVA). These findings underscore the general result that noise correlations are highest in shared field bins and lowest in one-field bins, with noise correlations in out of field bins being intermediate. The only contrast not obeying this general effect is the comparison between negative correlations of one-field bins and out of field bins for A1-A1 pairs, which did not reach significance $(p=0.10)$.

The strength of noise correlations was found to depend modestly on the area-based type of cell pair, with A1-A1 pairs showing stronger noise correlations than A1-V2L pairs (figure 3F, positive correlations: $\mathrm{A} 1-\mathrm{A} 1$ mean $=0.059, \mathrm{~A} 1-\mathrm{V} 2 \mathrm{~L}$ mean $=0.045, F(1,3.1)=22.6, \mathrm{p}=0.048$, negative correlations: $\mathrm{A} 1-\mathrm{A} 1$ mean $=-0.046, \mathrm{~A} 1-\mathrm{V} 2 \mathrm{~L}$ mean $=-0.038, F(1,5.2)=55.78, \mathrm{p}=0.002, \mathrm{ANOVA})$. Mean noise correlations for V2L-V2L cell pairs were intermediate to A1-A1 pairs and A1-V2L pairs, for both positive (V2L-V2L mean $=0.055)$ and negative correlations (V2L-V2L mean $=-0.040)$, and differences between V2L-V2L pairs and the other cell pairs were not significant (all $p>0.05$ ). There was no significant interaction between the type of bin and the composition of the neuronal pairs (positive correlations: $F(1,14587)=1.53, p=0.19$, negative correlations: $F(1,8312)=1.59, p=0.18$, ANOVA). We found qualitatively similar results when we defined location type not in terms of falling inside or outside of firing fields of the two units of a pair, but in terms of falling inside the $30 \%$ of bins with highest or lowest spiking activity of the two units of a pair.

To summarize, A1 and V2L populations do not only display similar distributions of firing fields across the maze, but pairs of $A 1$ and $V 2 L$ neurons also show fine-grained firing rate co-fluctuations which 
depend on both the similarity of location-selectivity of the units in a pair and the actual position occupied by the rat. Despite area-dependent differences in noise correlations, the similarity of A1-V2L noise correlation patterns to within-area co-fluctuations is rather striking.

\section{A1 activation precedes V2L activation in time}

230 The fine-grained co-fluctuations between $\mathrm{A} 1$ and V2L in the spatial domain raise the question how these 231 interactions are more precisely organized in time. The temporal firing relation between A1-V2L neuronal pairs was determined by computing the cross-correlograms over periods in which the rat was running on the track (running speed $>0.06 \mathrm{~m} / \mathrm{s}$ ). To correct for the impact that the forced unidirectional running may induce in estimating the temporal firing relation, cross-correlations were computed on the z-scored spiking activity after subtracting the mean activity in the associated spatial bin. Figure $3 \mathrm{G}$ shows the proportions of cross-correlation peaks of all A1-V2L pairs at different time lags averaged over rats. The narrow peak centered at -10 ms indicates that activation of A1 neurons mostly precedes V2L neuronal activity closely in time.

\section{Spiking activity carries information about position and head direction}

241 We next used information-theoretic measures to quantify the influence of navigation parameters, i.e.

242 position, running speed, head direction $\left(\theta_{\text {head }}\right)$ and changes in head direction $\left(\Delta \theta_{\text {head }}\right)$, on the spiking 243 activity of recorded units. Mutual information (MI) quantifies the reduction in uncertainty obtained about 244 spiking activity after observing these factors and captures both linear and nonlinear relationships (Lizier, 245 2014; Olcese et al., 2016). Of these individual factors, position carried the most information about spiking 246 activity of both $A 1$ and V2L units, followed by head direction (Fig. 4A; MI of position versus $\theta_{\text {head: }} A 1: p=$

$2471.1 * 10^{-15}$; V2L: $\mathrm{p}=3.0 * 10^{-15}$, Wilcoxon signed-rank test). Running speed and changes in head direction 248 carried relatively little information about spiking activity in both areas. For all individual factors mean $\mathrm{MI}$ 249 was higher in V2L than in $\mathrm{A} 1\left(\mathrm{p}=2.0 * 10^{-6}\right.$; running speed: $\mathrm{p}=4.7 * 10^{-4}, \theta_{\text {head: }}: \mathrm{p}=1.4 * 10^{-7}, \Delta \theta_{\text {head }}: \mathrm{p}=$ $2501.6 * 10^{-5}$, Mann-Whitney's U test).

251 When a particular $\theta_{\text {head }}$ is predominantly encountered at a given position, mutual information 252 cannot distinguish between the possibilities of a neuron encoding either that position, head direction or 253 a combination of the two. To determine the amount of information carried by spikes about speed, $\theta_{\text {head }}$ 254 and $\Delta \theta_{\text {head, }}$, that cannot be explained by (nonlinear) correlations with position, we computed the debiased 255 conditional mutual information (cMI) between each of the three factors conditional on position (Fig. 4B; 256 (Bos et al., 2019; Lizier, 2014)). Averaged across all spatially stable units, the amount of information that 
spike trains carried about running speed and changes in head direction beyond position (i.e. mean cMI(spikes, speed | position) and mean cMI(spikes, $\Delta \theta_{\text {head }}$ | position)) was negative for both $\mathrm{A} 1$ and $\mathrm{V} 2 \mathrm{~L}$, confirming that running speed and changes in head direction contributed little information about spiking activity on top of position (fig. 4B). In contrast, the average cMI for head direction, cMI(spikes, $\theta_{\text {head }}$ I position), was significantly larger than zero for both A1 and V2L (fig. 4B, A1: $p<10^{-15}$; V2L: $p<10^{-15}$; Wilcoxon's signed-rank test), indicating that spiking activity of both A1 and V2L units coded information about head direction in addition to information about position.

To study the prevalence of position coding in populations of single units, we calculated cMI between spikes and position conditional on $\theta_{\text {head, }}$ thereby excluding any contributions from $\theta_{\text {head }}$ (Fig. 4C). Significant cMI (spikes, position | $\theta_{\text {head }}$ ) was found in 140 out of 413 A1 units (33\%) and 197 out of 400 V2L units (49\%), indicating that spiking activity of large proportions of single units in both areas contains information on spatial position on top of any information on $\theta_{\text {head, }}$, with V2L showing a significantly larger proportion $\left(z=4.64, p=1.7 * 10^{-6}\right.$, binomial test; Fig. 4D). Smaller, but substantial subsets of neurons in each area showed significant cMI (spikes, $\theta_{\text {head }}$ | position), indicating that the spike trains of these neurons carry information about head direction even after correcting for position (fig. 4D, A1: 16.5\% and V2L: $31.2 \%)$, with also here $\mathrm{V} 2 \mathrm{~L}$ showing a significantly larger proportion of neurons $\left(z=4.9, \mathrm{p}=4.2 * 10^{-7}\right.$, binomial test). Among all units encoding position and/or head direction, V2L units were also significantly more likely to provide information on both factors (fig. 4D, A1: 35.1\%, V2L: 47.2\%, $z=2.33, p=0.010$, binomial test). In summary, this analysis confirmed that substantial fractions of A1 and particularly V2L neurons continued to show location- and head direction selectivity after correcting for the influence of the other behavioral covariate.

Position is the strongest predictor of A1 and V2 single unit activity, followed by head direction.

280 Next we used a random forest encoding algorithm (Benjamin et al., 2018) to determine how well spiking activity could be predicted by each of the behavioral factors. This provided the opportunity to extend the analysis of dependencies between behavioral factors and spiking activity beyond two behavioral factors. Encoding of firing rates of two units from $\mathrm{A} 1$ and $\mathrm{V} 2 \mathrm{~L}$ is exemplified in Figure 4E. For both areas, encoding quality was best for a model incorporating all predictors (Fig. 4F; all predictors vs. only position for A1 and V2L combined, $p<10^{-15}$, Wilcoxon's signed-rank test), whereas of the individual factors position provided the best performance (position vs. each other individual predictor for $\mathrm{A} 1$ and V2L, all $p<10^{-15}$, Wilcoxon's signed-rank tests). The large effect of position on encoding performance could not be explained by confounds arising from the other behavioral factors, since adding position to a model already utilizing all 
other factors led to substantial improvements in encoding performance for both A1 and V2L (fig. 4G; position vs. each other individual factor, all $\mathrm{p}<10^{-15}, \mathrm{~A} 1$ and $\mathrm{V} 2 \mathrm{~L}$ combined, Wilcoxon's signed-rank tests). Comparing the contributions of position and $\theta_{\text {head }}$ separately for individual units confirmed that among the behavioral factors considered, position was dominant in explaining spiking activity in both areas, while

$293 \theta_{\text {head }}$ provided the second strongest contribution (fig. 4G). This is also illustrated in Figure 4H, which shows

294 for each neuron the improvement in encoding when adding $\theta_{\text {head }}$ or position to models containing all other

295 behavioral factors. For the vast majority of units in both areas, adding either factor improved encoding quality, but this difference was larger for position than for $\theta_{\text {head. }}$ When analyzing the mean improvement in decoding performance achieved beyond all other predictors, we found that this value was larger for position (0.048) than for $\theta_{\text {head }}\left(0.019\right.$; all $p<10^{-15}$, Wilcoxon's signed-rank tests) or the other two covariates. Furthermore, the improvement due to $\theta_{\text {head }}$ was larger than for these other factors (speed: $0.011 ; p<10^{-15} ; \Delta \theta_{\text {head }}$ 0.008; $p<10^{-15}$, Wilcoxon's signed-rank tests).

Both the conditional mutual information and encoding analysis show that neurons can carry significant information on both factors. However, other factors such as stimulus availability, choice and reward may have influenced these results, because during track running they are closely associated with both position and head direction in space and/or time (Zaidel et al., 2017). Re-analysis of conditional information, now on data in which the period of stimulus presentation $\pm 1 \mathrm{~s}$ was eliminated, showed a decrease in the amount of information on $\theta_{\text {head }}$ conditional on position, which was nevertheless still significantly larger than 0 for both areas (cMI difference from $0, A 1: p=1.7 * 10^{-4}, \mathrm{~V} 2 \mathrm{~L}: \mathrm{p}<10^{-15}$, Wilcoxon signed rank tests; Supplementary Figure 2A, cf. Fig. 4B). Similarly, re-analysis of encoding improvement with data from which the stimulus presentation period $\pm 1 \mathrm{~s}$ was eliminated yielded similar results compared with the original finding (Supplementary Fig. 2B, cf. Fig. 4G). Together, these results suggest that position and, to a lesser degree, head direction are important drivers of firing activity of neurons in

312 A1 and V2L. However, we cannot rule out potential contributions to information related to position or 313 head direction as a result of correlations with additional subject-induced sensations (e.g. eye movements), 314 which we were unable to detect in the current dataset.

317 If the neuronal populations in $\mathrm{A} 1$ and $\mathrm{V} 2 \mathrm{~L}$ code the animal's track position, it should be possible to infer 318 the position of the rat from the collective neuronal activity. We used a Bayesian decoder to test whether 319 spatial location can indeed be predicted from population activity (sessions with $>16$ neurons/area were 320 included). Decoding of position was successful for both A1 and V2L, because the chance that a decoded 
position matched the true position of the rat was larger than the chance that it matched any other location on the track (i.e. clear diagonal structure in Fig. 5A, B; A1: $n=15$ sessions; V2L: $n=12$ sessions). The distributions of decoding errors, which are the Euclidean distances between the true and decoded positions, clustered near $0 \mathrm{~m}$ for both areas (Fig. 5C). V2L showed, on average, higher proportions of smaller errors than $\mathrm{A} 1$, indicating that decoding spatial position from $\mathrm{V} 2 \mathrm{~L}$ was more accurate than from A1 (V2L error: 0.11, 0.10 - 0.42; A1 error: 0.29, 0.26 - 0.37; median and interquartile range, in meters). error for both areas. We considered several population sizes, which were similar for both areas (A1: 1638 neurons, V2L: 16-40 neurons), but bootstrap analysis showed no signs of plateau performance with progressive population sizes (Fig. 5D). Although decoding from both areas likely could be improved had more neurons been recorded, at identical population size decoding performance for $V 2 L$ was better than for A1, both for the mean across sessions and for most individual sessions.

To investigate whether locations are encoded coherently across both areas, we calculated the correlation in instantaneous decoding error between A1 and V2L. Instead of only taking the magnitude of the error into account, errors in the $x$ and $y$ dimensions of the maze were considered separately and their directionality was preserved. When decoding errors were computed for sessions which contained $>16$ simultaneously recorded neurons in each area, we found significant correlations $(p<0.001$ for all 11 sessions). This was also the case when including all sessions where at least one of the two areas provided 16 neurons, again indicating that $\mathrm{A} 1$ and $\mathrm{V} 2 \mathrm{~L}$ encode locations coherently. However, these correlations may be confounded if certain locations are represented more accurately than others in both areas, or if

341 firing patterns are subjected to a common modulation by locomotion speed. To control for these 342 possibilities, we shuffled the data across time points at which the animal was in the same spatial bin and 343 running within the same range of speeds. The correlations between the shuffled decoding errors were 344 significantly lower than the observed correlations ( $x$-direction, $p=0.01, y$-direction $p=0.02$, Wilcoxon 345 signed-rank test). This difference remained significant when we included all sessions where at least one 346 area provided 16 neurons ( $x$-direction, $p=0.001$, $y$-direction, $p=0.01$ ). The residual decoding errors, 347 obtained by subtracting the shuffled distribution from the observed joint distributions, displayed a 348 diagonal structure (Fig. 5G-H), indicating that representations of location in A1 and V2L remain coherent 349 even when position cannot be decoded accurately from the population of either area. This coherency 350 exceeds what would be expected from a common influence of speed. In addition to the mean across 351 sessions (Fig. 5G-H), these results also held for individual rats (Supplementary Figure S3). It is unlikely that 352 error correlations can be accounted for by decoding artifacts in sessions with poor decoding performance 
because there was no evidence of a positive relationship between instantaneous decoding error correlations and average decoding error, rather their linear correlations were negative and not significant

355 (Supplementary Fig. 4).

\section{Discussion}

We showed location- and head direction-selective firing patterns in large proportions of neurons in A1 and V2L of freely moving rats navigating a figure-8 maze. This activity takes part in coherent representations across areas, as indicated by highly correlated firing-field densities (fig. 3A-C), correlated errors in reconstructed position (fig. 5) and cross-areal noise correlations which varied as a function of spatial tuning and immediate position (fig. 3D-F).

A first implication is that neural representations bound to subject location exist in the sensory cortex outside the visual system. Such activity may be expected in V2L because of similar observations in V1 (Fiser et al., 2016; Fournier et al., 2019; Haggerty and Ji, 2015; Ji and Wilson, 2007; Saleem et al., 2018) and egocentric trajectory correlates in spiking activity of posterior parietal cortex (Krumin et al., 2018; McNaughton et al., 1994; Nitz, 2006; Whitlock et al., 2012). These areas share anatomical borders with V2L and bidirectional, monosynaptic connectivity (Haggerty and Ji, 2015; Krumin et al., 2018; McNaughton et al., 1994; Miller and Vogt, 1984). That A1 neurons also show location-tuning is more surprising, because A1 activity has hitherto not been associated with animal location. However, in many mammals this area is required for sound localization (Heffner, 1978; Jenkins and Merzenich, 1984; Kavanagh and Kelly, 1987; Thompson and Cortez, 1983) and contains neurons tuned to the location of sound sources (Middlebrooks and Pettigrew, 1981; Town et al., 2017; Wang et al., 2019).

\section{Nature and function of location-selective firing in sensory cortices}

376 In addition to single or multiple peaks in single-cell firing rates tessellating the entire maze, we found that

377 A1 and V2L spike patterns were spatially stable across trials and coded significant amounts of information 378 on animal position (fig. 2 and 3). Our maze harbored repetitive elements requiring the same local 379 behavior, such as directional body turns or running along straight maze stretches, whereas many cells 380 showed single firing-rate peaks and thus did not reflect these repetitions (fig. 2). Furthermore, the 381 encoding analysis revealed animal position as the strongest predictor of A1 and V2L activity, even after correcting for head direction (fig. 4). Moreover, subject position could be inferred from A1 and V2L ensemble activity using Bayesian decoding (fig. 5). Despite these indications, we argue that neither our current findings, nor previous results on V1 (Fiser et al., 2016; Haggerty and Ji, 2015; Ji and Wilson, 2007; 
Saleem et al., 2018), necessarily imply coding of (allocentric) position per se, because proving this would require additional experimental manipulations to establish that location-selective activity is independent of the locomotion direction through a location and tolerates manipulation of local sensory cues (Knierim, 2002; Lansink et al., 2012; Leutgeb et al., 2005; Speakman and O'Keefe, 1990; Wilber et al., 2014).

We propose the more general alternative that sensory cortical areas integrate modality-specific evidence with information from other sensory, motor and association areas to generate sequential representations, which are not merely sensitive to local sensorimotor cues, but also to contextual elements (which may comprise spatial but also other task-relevant elements such as reward proximity, task rule execution, etc.). For instance, activation of visual cortex neurons depends on the subject's field of view, which in turn depends on animal position and head-direction (cf. Haggerty and Ji, 2015). This combination of view, position and head direction may give rise to a predictive visual representation in the cortex, which will be compared to further visual input to compute error signals, as posited by predictive processing models (Fiser et al., 2016; Friston, 2005; Keller and Mrsic-Flogel, 2018; Pennartz et al., 2019; Rao and Ballard, 1999). Although this proposal needs further testing, it is generally supported by the literature documenting extensive corticocortical connections (Bizley et al., 2007; Budinger and Scheich, 2009; D'Souza et al., 2016; Felleman and Van Essen, 1991; Gămănuţ et al., 2018; Harris et al., 2019;

401 Laramée et al., 2011; Leinweber et al., 2017), contributions to neural coding in sensory cortices by non402 sensory parameters (Goltstein et al., 2013; Namboodiri et al., 2015; Pakan et al., 2018; Shuler and Bear, 403 2006) and auditory-visual cortical interactions (Ibrahim et al., 2016; lurilli et al., 2012; Knöpfel et al., 2019; 404 Meijer et al., 2020; Meijer et al., 2017; Morrill and Hasenstaub, 2018).

This view does not conflict with a potential role for sensory cortices in updating spatial (e.g. hippocampal) representations in a bottom-up fashion, or with the navigational system contributing to top-down sensory predictions (Fournier et al., 2020). However, the hypothesis of the hippocampus 408 causally driving spatial coding in V1 (Saleem et al., 2018) faces the issue that the hippocampus proper 409 does not directly project to the sensory cortices, and its output is transformed by the synaptic matrices of 410 intermediate parahippocampal regions on which also non-hippocampal structures converge (Furtak et al., 411 2007; Rusu and Pennartz, 2020; Witter et al., 2000). Instead of guiding spatial navigation, the bidirectional, 412 cortico-hippocampal circuitry may subserve declarative memory consolidation (Eichenbaum, 2000; 413 McGaugh, 2000; O'Keefe and Nadel, 1978; Rusu and Pennartz, 2020; Squire, 1986). In this process, 414 distributed neocortical activity selective for parts of a behavioral sequence may form a common 'pointer' 415 or marker for binding together cross-modal information (Teyler and DiScenna, 1985). 


\section{Sensitivity to head direction in A1 and V2L}

418 For $33 \%$ of neurons in $\mathrm{A} 1$ and $49 \%$ in V2L, the information conveyed on head direction was significant 419 after correcting for position (fig. 4B, 4D). As for subject location, this finding relates to how sensory420 specific states coded by $\mathrm{A} 1$ and $\mathrm{V} 2 \mathrm{~L}$ depend on head direction, or how these sensory cortices may use this 421 sensitivity to emit head direction signals to target areas. Head direction signaling is regulated by the 422 vestibular nuclei carrying information about the head's motion relative to external space (Cullen, 2014) 423 and, in rodents, about static neck position (Barresi et al., 2013; Medrea and Cullen, 2013). Vestibular input 424 is a key contributor to the brain's head-direction system (Taube, 2007), including anterior thalamus 425 (Taube, 1995), post-subiculum (Taube et al., 1990) and medial entorhinal cortex (Sargolini et al., 2006). 426 Vestibular information was shown to reach the visual system directly and indirectly, viz. via the 427 retrosplenial cortex (Vélez-Fort et al., 2018), which was proposed to mediate between the sensory 428 cortices and the head direction system of the temporal lobe (Page and Jeffery, 2018). Whether head429 direction signaling in A1 and V2L has a causal role in distributing head-direction information to target 430 areas is up for further research. The collective evidence supports the hypothesis that areas along the 431 cortical hierarchy may use both allocentric and egocentric representations, with a gradient of egocentric432 to-allocentric processing from sensory to temporal cortices, as also proposed for parietal-retrosplenial 433 circuitry, where V2L is sometimes included as part of parietal cortex (Chen et al., 2018; Clark et al., 2018; 434 Wilber et al., 2014).

\section{Comparison between primary auditory cortex and secondary visual cortex}

437 We were particularly struck by the broad, qualitative similarities between A1 and V2L spiking patterns. 438 Both areas showed comparable levels of spatial stability, amounts of firing fields per unit (fig. 2), spatial 439 distributions of firing fields and noise correlations (fig. 3). Moreover, the contributions to predictions of 440 firing-rate patterns from position, head direction and other factors were highly similar for A1 and V2L (fig. 441 4). These observations lend support to the hypothesis that coordinated representations are a general 442 feature of sensory cortical areas.

$443 \quad$ Neural coding in A1 and V2L also showed interesting quantitative differences, which consistently 444 point to a higher spatial information content (fig. 2), stronger correlations with location and head direction 445 (fig. 4) and better position reconstruction in V2L than A1. How this greater accuracy arises in V2L is 446 unknown, but it may relate to a larger amount of spatially informative visual cues in our maze compared 447 to auditory cues, and to more consistent changes in visual inputs due to self motion than to (self-induced) 448 auditory inputs. In view of this greater accuracy, it is interesting to observe the slightly earlier firing in A1 
than V2L units (fig. 3G). This might reflect the exploitation of potentially faster, subcortical processing of auditory compared to visual information (Picton et al., 1974).

\section{Significance of cross-areal coordination in cortical mapping of location and head direction}

453 Arguably our most novel result is that location-selective representations are highly coherent across

454 sensory domains of the cortex (fig. 3, 5), suggesting that location- and head direction-sensitive mappings

455 in auditory and visual cortical systems are not computed independently but are coordinated. Such

456 coordination of context-dependent sensory mappings offers the computational advantage that evidence

457 from multiple sensory modalities can be combined to improve estimates of the subject's task-relevant

458 state, although this comes at the expense of error sharing.

459 Previous studies on auditory-visual interactions were predominantly guided by the theoretical

460 framework of multisensory cue integration, whereby evidence for the detection of a stimulus in one

461 modality is augmented by another modality (Fetsch et al., 2013; Meijer et al., 2019; Stein et al., 2014).

462 Our findings go beyond integration of discrete sensory cues and instantaneous sensory states as they

463 indicate a cross-modal coordination of context-sensitive representations. In our proposal this common

464 mapping subserves the construction of a multimodal survey of the subject's current situation, thereby

465 enabling efficient goal-directed action planning and execution (Pennartz, 2018). As argued by Hawkins et

466 al. (2017), self-parameters including head direction and position within a task sequence are key priors in

467 determining how and when to undertake goal-directed actions. In line with predictive processing,

468 knowledge of these self-parameters is required to interpret novel sensory inputs and anticipate sensory

469 outcomes of actions (cf. Schürmann et al., 2019). 


\section{Materials and Methods}

\section{Experimental design}

473 Subjects

474 Experiments were performed on Lister Hooded rats ( $n=3$, Envigo, the Netherlands) at an age between 9 475 and 40 weeks. All rats were socially housed during behavioral training, but individually housed during 476 periods of recordings when rats had an implanted tetrode-array, under a normal day/night cycle (lights 477 on: 8:00am, lights off: 8:00pm). The rat's food intake was restricted such that its weight was at least 85\% 478 relative to the standard growth curve provided by the breeder (Envigo, the Netherlands), corrected for 479 the deviance in weight between the rat and the curve in the week before the start of food restriction. 480 Weights were maintained at a stable level after rats reached healthy, adult body weight (Clemens et al., 481 2014; Newby et al., 1990). Rats had ad libitum access to water throughout the experiment. All experiments 482 were performed in accordance with the National Guidelines on Animal Experiments and were approved by the Animal Experimentation Committee of the University of Amsterdam.

\section{Behavioral setup}

486 Rats were trained to discriminate between auditory and/or visual stimuli on an automated, rectangular figure- 8 shaped track $(92 \mathrm{~cm} \times 73 \mathrm{~cm}$ ) which was raised $55 \mathrm{~cm}$ off the ground (fig. 1A). The track's alleys were made of black painted aluminum (width $=8.7 \mathrm{~cm}$ ) and contained raised edges $(1.0 \mathrm{~cm}$ ). At the front of the track, two LCD monitors (liyama ProLite B2776HDS) and two audio speakers (Audaphon Neo CD 3.0) were available for stimulus presentation. The monitors were positioned symmetrically from the center of the track and the speakers were located above the top-left and top-right corners of the left and right monitors, respectively. During early training stages, transparent polycarbonate walls lined the central alley to prevent the rat from prematurely exiting this alley. Additionally, two transparent polycarbonate sliding doors were positioned at the front and back of the central alley. The walls and door at the front of the central alley contained small holes to allow for perception of the auditory stimuli. Two reward wells were positioned at the left and right edges of the track's front alley. An additional reward well was positioned in the central alley, towards the front-end T-junction. Fluid sucrose solution (15\% in tap water) was delivered to the wells by syringe pumps (Razel, VT, USA). All reward wells contained infrared photodetectors to detect nose pokes and licks. The motor activity of the rat on the track was registered with additional photodetectors, located near the T-junctions at the front and back ends. 
behavioral events and neuronal activity patterns. It was positioned in an enclosure of black curtains ( 2.8 $x 2.2 \mathrm{~m}$ ) within a sound-attenuated room of $3 \times 3 \mathrm{~m}$. The room was dimly lit by a small LED light pointed

505 towards the ceiling. The experimenter observed training and experiments from an adjacent room to minimize interference with the rats' behavior.

Stimuli

509 On each trial of the behavioral task, rats had to discriminate between a target stimulus, displayed on one of the screens and/or the adjacent speaker, and a distractor stimulus, appearing on the other screen and/or speaker (Fig. 1B). Target and distractor were either unisensory (visual or auditory) or multisensory

512 (audiovisual) and differed in stimulus amplitude. Three types of target/distractor combinations were in 513 equal proportions presented to the rat; large-difference unisensory trials (1/3), small difference 514 unisensory trials (1/3) and small difference cross-modal trials (1/3). In a separate session for each rat, the 515 threshold amplitude differences (i.e. the amplitude differences at which the rat shows a correct response 516 in $50 \%$ of the stimulus presentations) were determined using a staircase procedure. On the basis of this 517 data, stimulus parameters were set for each rat such that the discrimination performance for the large518 difference unisensory trials was $>70 \%$ correct and for the small-difference unisensory trials was $>50 \%$ correct. The stimulus settings remained the same for all recording sessions of a rat. The amplitude differences for the stimulus components in the audiovisual trials were identical to the differences for the small-difference unimodal stimuli of the same session. The specific screens (left or right) at which target and distractor were displayed were pseudorandomly selected for each trial, such that the target stimulus was never displayed more than four successive trials at the same side and the difference in left and right target-presentations across the session was not more than 4.

Visual stimuli were full-screen, upward-moving, black-and-white checkerboards $(0.1$ 526 cycles/degree, 4 cycles/second; fig. 1B). When no visual stimuli were displayed, a grey background was visible. All visual stimuli and the background were gamma corrected and had the same overall luminance as measured with a photometer. Contrast values between light and dark checkers varied between 0 and 1 , in which 0 indicates no contrast and 1 indicates the maximum contrast possible with the monitor at its lowest brightness setting.

Auditory stimuli were composed of white noise, which was band-passed between 10 and $25 \mathrm{KHz}$. When auditory stimuli were absent, background noise was played. Background noise was white noise 533 band-passed between 8 and $12 \mathrm{KHz}$. For auditory stimuli, the difference between target and distractor 534 was in the relative volume between the speakers. Relative auditory volume ranges between values of 0 
535 and 1 , with 0 and 1 indicating that the volume is fully accounted for by one or the other speaker.

536 Background noise was played with a contrast of 0.5 , i.e. identical volume through both speakers.

537 Therefore, at every moment during the session, sound was playing at the same volume, which was set to

$53876 \mathrm{~dB}$ at the central reward well.

Behavioral training

541 After rats had learned to complete unidirectional laps on the track and to nose-poke for a duration of 1

542 second to earn reward, they were trained to discriminate between target and distractor stimuli and to

543 respond by choosing the side at which the target stimulus was present. When the front door opened at

544 the start of the trial, the target visual stimulus was presented on one screen (i.e. on one side of the track)

545 while the other screen maintained the grey background. The rat earned a reward if it poked in the well at

546 the side of the track corresponding to where the stimulus was displayed. The stimulus presentation lasted

547 until 3 seconds following reward delivery, with the aim of strengthening the stimulus-response-outcome

548 association. When the rat made a nose poke at the incorrect side, stimulus presentation stopped

549 immediately. From this stage onwards, the length of the nose pokes to start stimulus presentation was

550 increased incrementally from 0 to $0.5-1.5$ seconds (randomized across trials). If the rat performed at least

55160 trials within 60 minutes with $70 \%$ correct trials on three out of five consecutive days, stimuli switched

552 from visual to auditory. If this criterion was met also for auditory stimuli, subsequent sessions included

553 audio, visual stimuli and audio-visual stimuli in equal proportions and presented according to the

554 pseudorandom schedule (see Stimuli). In multisensory trials, the auditory and visual stimulus components

555 were always presented on the same side of the track; i.e. no sensory conflicts were created. Once the rat

556 reached the same criterion with these three trials types included in the session, the discrimination

557 problem was made progressively more difficult, by introducing distractor stimuli and lowering the

558 contrast of visual target stimuli in subsequent sessions. The difference in amplitude between target and

559 distractor stimuli was gradually decreased over training sessions but never below the level described

560 above for 'low-difference stimuli'. In parallel with the increase in difficulty, the display time of the stimuli

561 was progressively shortened until the stimulus duration was 2-3 s. To prevent rats from developing

562 habitual or stereotyped response preferences, extra sessions were occasionally included in the training.

563 In these sessions, rats were allowed to collect reward from the correct well after sampling the incorrect

564 well. After an incorrect nose poke, trials continued until the correct well was sampled. These sessions did

565 not count towards criterion performance. 
In the final task, the trial procedure was similar to training stage 4 and now the small and large difference unimodal stimuli were presented alongside with the crossmodal stimuli (see Stimuli). To ensure enough trials were performed in all conditions to allow statistical comparison, each session contained either visual or auditory unimodal trials, in addition to multisensory trials. Recordings commenced when rats consistently performed at criterion level: $>60$ trials in 60 minutes with $>70 \%$ correct on large-difference unimodal stimuli and above chance level for small difference unimodal stimuli and multisensory stimuli.

\section{Tetrode array and surgical procedure}

575 A custom-made, 128 channel tetrode-array was implanted over the right hemisphere of three rats (Bos et 576 al., 2017; Lansink et al., 2007). Four bundles of 8 individually moveable tetrodes each were targeted to primary auditory cortex (A1, -4.4 mm AP, $6.8 \mathrm{~mm} \mathrm{ML,} \mathrm{-4.4} \mathrm{mm} \mathrm{DV),} \mathrm{lateral} \mathrm{secondary} \mathrm{visual} \mathrm{cortex} \mathrm{(V2L,} \mathrm{-}$ $5.8 \mathrm{~mm}$ AP, $6.0 \mathrm{~mm} \mathrm{ML},-2.6 \mathrm{~mm} \mathrm{DV}$ ), hippocampal area CA1 (CA1, -3.6 mm AP, $2.4 \mathrm{~mm} \mathrm{ML,} \mathrm{-2.6} \mathrm{mm} \mathrm{DV)}$ and perirhinal cortex area 36 (PRH, -4.4 mm AP, $6.8 \mathrm{~mm} \mathrm{ML,} \mathrm{-7.0} \mathrm{mm} \mathrm{DV).} \mathrm{Hippocampal} \mathrm{and} \mathrm{perirhinal}$ data were not used in the current study. Thirty minutes before surgery, rats received the analgesics meloxicam (Metacam, $2 \mathrm{mg} / \mathrm{kg}$ ) and buprenorphine (Buprecare, $0.04 \mathrm{mg} / \mathrm{kg}$ ) subcutaneously, as well as the antibiotic enrofloxacin (Baytril, $5 \mathrm{mg} / \mathrm{kg}$ ). Anesthesia was induced using $3 \%$ isoflurane in oxygen and maintained with 1-2\% isoflurane. Animals were mounted in a stereotaxic device (Kopf; Tujunga, CA, USA) and placed on a heating pad to maintain their body temperature. A single craniotomy was made such that the points at which the bundles entered the brain were positioned relative to bregma at $-5.4 \mathrm{~mm} \mathrm{AP}$ and $2.8 \mathrm{~mm} \mathrm{ML} \mathrm{(A1),}-5.9 \mathrm{~mm} \mathrm{AP}$ and $4.0 \mathrm{~mm} \mathrm{ML} \mathrm{(V2L),} \mathrm{-3.6} \mathrm{mm} \mathrm{AP} \mathrm{and} 1.7 \mathrm{~mm} \mathrm{ML} \mathrm{(CA1)} \mathrm{and}-3.5 \mathrm{~mm} \mathrm{AP}$ and $3.8 \mathrm{~mm} \mathrm{ML} \mathrm{(PRH).} \mathrm{Six} \mathrm{screws} \mathrm{were} \mathrm{placed} \mathrm{into} \mathrm{the} \mathrm{skull,} \mathrm{with} \mathrm{the} \mathrm{screw} \mathrm{positioned} \mathrm{over} \mathrm{the} \mathrm{frontal}$ bone serving as electrical ground for the tetrode array. The hyperdrive was positioned such that the bottom of the bundles touched the cortical surface. The craniotomy was then sealed using silicone adhesive (Kwik-Sil) and dental cement was used to fix the hyperdrive and screws to the skull. Postoperative care consisted of subcutaneous injections of the analgesic meloxicam (2 days) and Baytril antibiotic (1 day) and application of wound healing ointment (Acederm, Ecuphar, Breda, Netherlands). On

593 post-operative days 1-3, rats received $10 \mathrm{~g}$ of extra food softened in water to facilitate consumption. 594 Tetrodes were gradually lowered to their target regions across the first week after surgery. During recordings their depth was estimated from the number of turns to the guiding screws and from the online Local Field Potential (LFP) profiles. 
599 Spikes and LFPs were recorded using tetrodes (Gray et al., 1995)(nichrome, California Fine Wire, $16 \mu$ per 600 lead, gold-plated to an impedance of 500-800 KOhm) using a Neuralynx Digitalynx SX recording system

601 (Neuralynx, Bozeman MT). Raw signals were buffered using four 32-channel unity-gain head stage 602 amplifiers before being passed through an automated commutator (Neuralynx, MN). Each of the four 603 tetrode bundles contained an additional electrode that served as a reference channel and which was 604 positioned in the white matter near the tetrode bundle. The recorded signals were the raw signals with 605 the reference signal subtracted. For spike recordings, signals were band pass filtered between 600 and $6000 \mathrm{~Hz}$. Putative spikes were recorded for $1 \mathrm{~ms}(16 \mathrm{bit}, 32 \mathrm{KHz})$ from all leads of a tetrode whenever the signal on any lead of that tetrode crossed a predefined threshold. LFPs were low-pass filtered below 300

$608 \mathrm{~Hz}$ and recorded continuously (16 bit, $3.2 \mathrm{KHz}$ ). The behavior of the rat was tracked by a ceiling-mounted camera (at 720 by 576 pixels, $25 \mathrm{fps}$ ) and timestamped by the Digitalynx SX.

612 used for clustering were energy, the first derivative of the energy, the overall peak height and the peak 613 height during samples 6-11 where the action potential peak is expected. Clusters were included for further 614 analysis based on a combination of quality metrics (Schmitzer-Torbert et al., 2005): L-ratio $(<0.2-0.8)$, 615 isolation distance $(>15-24)$ and interspike interval $(1.2 \mathrm{~ms})$ violations $(<0.1 \%-0.5 \%)$.

Histology

618 Following the last recording session animals were anesthetized using Isoflurane and electrolytic lesions 619 were made at each tetrode tip by passing current (18 uA for $2 \mathrm{~s}$ ) through two leads of each tetrode. At 620 least twenty-four hours later, the animal was deeply anesthetized with an intraperitoneal injection of 621 Nembutal ( $1.0 \mathrm{ml}$, sodium pentobarbital, $60 \mathrm{mg} / \mathrm{ml}$, Ceva Sante Animale, Maassluis, Netherlands) and 622 transcardially perfused with $0.9 \% \mathrm{NaCl}$ solution, followed by perfusion of a $4 \%$ paraformaldehyde solution 623 (pH 7.4 phosphate buffered). The brain was extracted and placed in 4\% paraformaldehyde solution for at 624 least 24 hours post-fixation, after which 40 um transverse sections were made using a vibratome. These 625 were stained with Cresyl Violet which allowed reconstruction of tetrode tracks and their endpoints 626 marked by electrolytic lesions (Paxinos and Watson, 2007)(Supplementary fig. 1). Because it was not 627 possible to determine with absolute certainty how each endpoint corresponded to the identity of 628 individual tetrodes, the considered neuronal pools likely contain a small subset of units recorded in the 629 vicinity of the target locations. Based on the average number of neurons included from the respective 
recording sessions for $\mathrm{A} 1$ and $\mathrm{V} 2 \mathrm{~L}$, the estimated number of neurons recorded outside their target areas was 16 for V2L and 32 for A1.

\section{Statistical Analysis}

\section{Statistical procedures}

635 Unless specified otherwise all statistics were performed using linear mixed models (LMMs) or generalized 636 linear mixed models (GLMMs) in MatLab (MathWorks, Natick, MA), depending on the distribution of the 637 data. All reported statistical quantities (group means, regression slopes, confidence intervals etc.) were 638 derived from the (G)LMMs. All reported confidence intervals are $95 \%$ prediction intervals. To estimate the 639 denominator degrees of freedom (DF2) for F-tests, the Satterthwaite approximation was used for LMMs 640 and the residual degrees of freedom for GLMMs. To correct for multiple comparisons, $p$-values are 641 adjusted using the Holm-Bonferroni method where applicable $\{$ Holm, $1979 \# 172\}$ (fig. 2H, 3E-F).

\section{Inclusion criteria}

644 Behavioral performance was measured as the percentage of correct responses to stimulus presentation 645 in a session. Sessions were included for analysis only if the performance was above $95 \%$ of a distribution 646 of performance expected from sessions with an equal number of trials and chance performance $(50 \%$ 647 correct trials). Additionally, to exclude sessions where animals had a preference for a particular response 648 direction, sessions were included only when responses to both left and right stimuli were performed 649 above chance using the same procedure.

\section{Z-scored spiking activity and firing rates}

652 Spike trains of individual units were binned into $1 \mathrm{~ms}$ temporal bins and smoothed with an exponential 653 window with a time constant of $150 \mathrm{~ms}$. Z-scored spiking activity was calculated by subtracting the mean 654 from the smoothed spike train and dividing the result by the standard deviation.

657 The location of the rat's body and head were determined separately for each frame of recorded video 658 using custom scripts made with Bonsai Editor (Lopes et al., 2015). Frames with erroneously assigned 659 positions were manually corrected. The raw tracking data was smoothed using the smooth() function in 660 Matlab using the 'rlowess' method and a span of 5 pixels. Head direction was determined for each video 661 frame as the angle of the body-head axis of the rat with respect to the left-to-right axis of the setup. 
663 Rate maps were constructed in 2D by spatially binning (bin size $=10$ by 10 pixels or $4.2 \mathrm{~cm}^{2}$ ) the smoothed 664 spike trains of single units into the spatial bin occupied by the rat in each videoframe, summing the firing rates per bin and then averaging over the time spent in each bin (occupancy). Binned spike trains and occupancy maps were independently smoothed by convolution with a 2D Gaussian (std = 1 bin) before averaging. Time segments in which the running velocity of the rat was $<6 \mathrm{~cm} / \mathrm{s}$ were excluded.

\section{Linearization of position data and $1 D$ rate maps}

670 The goal of linearizing position data is to allow more powerful analyses relating localized spiking activity

671 to behavior. The lateral range of body motion on the track was limited, and the firing fields observed on

672 the 2D rate maps generally spanned the full width of the track. Linearization therefore allows to focus on

673 the spatial dimension containing the majority of the rate map structure. Linearization of the rat's position

674 was achieved by first determining the average path of the rat across the setup for each recording session.

675 This was achieved by manually tracing the locations with highest occupancy of each session's occupancy 676 map. The starting point of the linearized track was chosen as the starting point of the right alley segment at the three-way junction at the front of the setup (FC; fig. 1A). The linearized track then consisted, in this order, of the right side of the track, central alley, left side of the track. The end position was the center of the back-alley segment at the three-way junction. The linear position was then determined as the point along this linearized track closest to each observed 2D position. Raw linearized position was smoothed by convolution with a Gaussian with standard deviation of 2 pixels $(0.4 \mathrm{~cm})$. Linear speed was then calculated from the linear location using the gradient() function in MatLab, and linear acceleration was calculated similarly from linear speed. Finally, because the linearized track length varied slightly between rats, linear

684 location was normalized to the mean length across animals $(334 \mathrm{~cm})$. Linear rate maps were made 685 similarly to the $2 \mathrm{D}$ rate maps but using the linear position data, using the same inclusion criteria and 686 smoothing parameters and with a bin size of 16 pixels $(3.3 \mathrm{~cm})$. Joint rate maps (fig. 3A-B) were 687 constructed from the linear rate maps of each neuron by normalizing each rate map between 0 and 1 , 688 and then sorting all rate maps of all sessions and rats by the location of the peak firing rate (dark red in 689 fig. 3A).

692 The procedure for determining the location of firing fields was similar to the method described by 693 (Haggerty and Ji, 2015), using the unit's linear rate map as a basis. First the rate map was smoothed using 
694 the smooth() function in Matlab (MathWorks, Natick, MA) with the 'rlowess' method and a span of 5 bins.

695 The baseline firing rate was determined as the $40^{\text {th }}$ percentile of this smoothed rate map. The baseline 696 was subtracted from the rate map and local maxima were determined for the baseline-corrected rate 697 map. Local maxima were kept for further processing if the rate was $>1 \mathrm{~Hz}$ and $>0.2 x$ the baseline rate.

698 Field boundaries were determined as the bins nearest to a peak where the firing rate was $<10 \%$ of the 699 peak firing rate. This procedure sometimes produced very small fields near or on the slope of larger fields 700 ('shoulders'). Such shoulders were discarded if the border of the field was two or fewer bins apart $701(<=6.6 \mathrm{~cm})$ from the border of a taller field, unless it was two or more bins $(>=6.6 \mathrm{~cm})$ away from its own 702 border and the peak was $>1.5$ times the value at this border. After the removal of spurious small fields, 703 the borders of remaining fields were extended to the bins where the activity fell below the peak cut-off 704 of $1 \mathrm{~Hz}$ and 0.2 times the baseline rate, or until they reached the border of another peak. This procedure 705 was followed for each single unit from the tallest to the lowest peak. Single fields that spanned the T706 junctions at the front and back of the experimental track were prevented from being detected as two 707 separate fields by considering the bins of the linear rate maps that border the T-junctions as adjacent.

Spatial Stability

710 The spatial stability of the localized firing of individual units was determined using a permutation analysis.

711 First, separate linear rate maps of each individual trial were constructed, similarly to the procedure 712 described above, except that the data was split into leftward and rightward trials. The correlation between 713 a single-trial rate map and the rate maps of all other trials on the same side of the track was computed 714 (Pearson correlation), for each trial and then the average correlation coefficient was computed. These 715 steps were repeated for 1000 shuffled versions of the spiking data. For each shuffled iteration, the spiking 716 data was temporally rotated within a single trial by a random number of samples (Louie and Wilson, 2001). 717 This method of shuffling leaves the temporal structure of spiking patterns largely intact. Positions and 718 spikes emitted during periods of immobility $(<0.06 \mathrm{~cm} / \mathrm{s})$ were excluded from analysis. A unit was 719 considered spatially stable if its average single-trial rate map correlation, for both left- and rightward laps, 720 was higher than $95 \%$ of the distribution of average, shuffled single-trial rate map correlations of the 721 corresponding side. To calculate the spatial stability index, the mean of the distribution of average, 722 shuffled single-trial rate map correlations was subtracted from the average observed single-trial rate map 723 correlation; the result was divided by the standard deviation of the shuffled distribution. This procedure 724 was done for each unit for both left- and rightward trials and the reported spatial stability index was the 725 mean of those two. 
$727 g\left(P_{\text {stable }}\right)=\ln \left(P_{\text {stable }}\right)$ and the equation:

728

$$
P_{\text {stable }} \sim \text { Area }+(1+\text { Area } \mid \text { Subject })
$$

Where Area is a categorical variable indicating the cortical area where the unit was recorded and Subject

732 is a categorical variable indicating from which experimental subject the data originated.

734 Spatial information

735 Spatial information (SI) for each unit was calculated from the linearized location data and firing rate

736 following Skaggs et al. (1992):

$$
S I=\sum_{i=1}^{N} P_{i} \frac{X_{i}}{r} \log _{2} \frac{X_{i}}{r}
$$

Where $P_{i}$ is the probability of finding the animal in bin $i, X_{i}$ is the sum of the firing rates observed when the animal was found in bin $i, r$ is the mean spiking activity of the neuron and $N$ is the number of bins of the linearized trajectory (104). The SI for each unit was modeled with a GLMM using a gamma distribution with link function $g(S I)=S I^{-0.01}$ and the equation:

$$
S I \sim \text { Area }+(1+\text { Area|Subject })
$$

\section{Correlating firing field densities}

746 Firing field densities were calculated per rat by first determining for each linear spatial bin the number of

747 firing fields, across all units, in which this bin took part. Then this number was divided by the subject's 748 total number of fields. The average firing field density is reported as the mean of the field densities for 749 the individual rats. Firing field densities of $A 1$ and V2L were correlated with an LMM using the equation:

$$
\text { Density }_{A 1} \sim \text { Density }_{V 2 L}+\left(1+\text { Density }_{V 2 L} \mid \text { Subject }\right)
$$

754 Linear regression was used to determine whether firing field correlations between $\mathrm{A} 1$ and V2L could be 755 explained by similar linear dependencies between single unit firing rates and behavioral covariates. The 
instantaneous, z-scored spiking activity of each unit was regressed on linear running speed, acceleration, head direction and change in head direction (angular velocity) using the general linear model:

$$
Z \sim \operatorname{Spd}+A c c+\operatorname{Sin}_{H D}+\operatorname{Cos}_{H D}+\omega_{H D}
$$

Where $Z$ is the instantaneous, z-scored spiking activity, $S p d$ is linear speed, Acc is linear acceleration, $\operatorname{Sin}_{H D}$ and $\operatorname{Cos}_{H D}$ are the sine and cosine of head direction and $\omega_{H D}$ is the angular velocity in head direction. The model residuals of each unit were used to produce linear rate maps and subsequently determine firing fields without linear dependencies on the behavioral covariates.

Stimulus responsiveness

The responsiveness of a unit to the sensory stimuli was assessed by statistically comparing the mean, zscored firing rates of the time intervals of [350 - 50] ms pre-stimulus onset and [0-300] ms post-stimulus onset using Wilcoxon's signed-rank test (alpha $=0.05$ ). A subset of units gradually increased or decreased firing rates before stimulus onset ("ramping activity"), without showing a change in spiking activity at stimulus onset. To preclude that such activity would erroneously be considered as significantly stimulus responsive, only units with a stable pre-stimulus onset firing rate were considered; i.e. the unit's firing rates between [1000-700 ms] and [350-50] ms pre-stimulus onset were required to be similar (Wilcoxon signed rank test, $\mathrm{P}>0.05)$. The probability of a unit's responsiveness to stimuli was modelled using a GLMM with link function $g\left(P_{\text {resp }}\right)=\ln \left(P_{\text {resp }}\right)$ and equation:

$$
P_{\text {resp }} \sim \text { Area } * \text { Modality }+(1+\text { Area }+ \text { Modality } \mid \text { Subject })
$$

Where Modality is a categorical variable indicating the modality (auditory or visual) of the stimulus being considered.

\section{Noise correlations}

783 Rate map similarity between neurons was first determined for each pair of units as the Pearson correlation coefficient of their linear rate maps. To determine noise correlations, we first temporally binned the instantaneous, z-scored spiking activity into $10 \mathrm{~ms}$ bins and then subtracted from that the mean spiking activity at the linear spatial bin occupied by the rat at each instant. This difference constitutes

787 instantaneous variability in spiking activity not predicted by spatial position. Additionally, for each pair of 
units we categorized locations along the track as i) occurring in a firing field of both units ("shared field bin"), ii) occurring in a firing field of only one of the units ("one-field bin") or iii) occurring outside of firing fields of both units ("out of field bin"). For each pair of units we calculated noise correlations as the Pearson correlation coefficient of the instantaneous variability of the activity of two units, and we did so separately for each of the three bin types.

Analysis proceeded with pairs showing significant noise correlations, determined via comparison

794 to shuffled distributions of these correlations. These distributions were made by computing noise correlations after shuffling samples of spiking activity within the same linear spatial bin and repeating this procedure 5000 times. A pair was considered significantly correlated if its actual correlation exceeded $99 \%$ of the shuffled distribution for at least one of the three location types.

For significantly correlated pairs, mean noise correlations across location types, were regressed on their rate map correlations with an LMM using the equation:

$$
R_{M N} \sim R_{R M} * \text { Type }_{\text {Pair }}+\left(1+R_{R M}+\text { Type }_{\text {pair }} \mid \text { Subject }\right)
$$

Where $R_{M N}$ is the mean noise correlation of a pair across location types, $R_{R M}$ is the rate map correlation and Type Pair is the type of pair (A1-A1, V2L-V2L or A1-V2L). Here we used the mean noise correlation across location types rather than the overall noise correlation since the latter suffers from sampling bias if noise correlations for different location types are not equal, because pairs with high rate map correlations contribute more samples from shared field bins and out of field bins than from one-field bins. the equation:

812 Where $R_{\text {noise }}$ is the average noise correlation for a unit pair at one of the three location types and Type bin $_{\text {. }}$

813 is a categorical variable indicating the location type.

\section{Cross-correlogram}

816 Cross-correlations of A1 - V2L unit pairs were computed from the z-scored spiking activity of all spatially 817 stable units, during periods with running speed $>0.06 \mathrm{~m} / \mathrm{s}$. Spiking activity was first binned in $10 \mathrm{~ms}$ bins. 818 The mean spiking activity observed in the spatial bin occupied by the rat at each instant was then 819 subtracted from the spiking activity in order to prevent the relative spatial positions of firing fields of two 
820 units from biasing the shape of the cross-correlogram. In doing so, the cross-correlation only considered

821 activity fluctuations with respect to the mean activity observed at each location. Histograms were made

822 of the distribution of cross-correlogram peak lags for each animal individually, which were then averaged

823 to produce the histogram shown in fig. $3 \mathrm{G}$.

824

825 Information theoretic analysis

826 For the information theory-based analyses, linear spatial bins of $\sim 16.4 \mathrm{~cm}$ were used, such that the

827 linearized track was divided into 21 bins. As predictors we used running speed, head direction ( $\left.\theta_{\text {head }}\right)$ and

828 head direction change $\left(\Delta \theta_{\text {head }}\right)$. Running speed and $\theta_{\text {head }}$ were calculated as described above, and $\Delta \theta_{\text {head }}$

829 was calculated as the degrees per second change in $\theta_{\text {head. }}$. The predictors were binned in 21 equipopulated

830 bins to match the number of location bins. Spike counts were binned in 300 ms time bins, and instances

831 with running speed $<0.1 \mathrm{~m} / \mathrm{s}$ were excluded. Discrete mutual information (MI) and discrete conditional

832 mutual information (CMI) were computed using the Java Information Dynamics Toolkit (JIDT)(Lizier, 2014).

833 Bias due to finite sample sizes was corrected by generating for every computation of $\mathrm{Ml}$ and $\mathrm{cMl}$ a

834 population of 500 surrogates. When creating the surrogates, only spike count vectors were shuffled, such

835 that the relationship between the target and conditional predictor (in the case of cMI) was preserved. The

$836 \mathrm{MI}$ and $\mathrm{CMI}$ values reported are the differences between the observed values and the average of the

837 surrogate populations. Differences in MI between A1 and V2L were statistically assessed using Mann-

838 Whitney's $U$ test. The p-value of $\mathrm{cMI}$ values of individual units was computed as the fraction of the

839 surrogate dataset which had information values higher than the observed one. To correct for multiple

840 comparisons, a Bonferroni correction was applied across all neurons and tested at the 0.05 significance

841 level. Reported confidence bounds correspond to 95\% bootstrap confidence intervals, computed with the

842 Seaborn Python library (v0.9.0, 2018).

Encoding: predicting spike trains from behavioral variables

845 First, the position of the rat was taken as the two-dimensional variable with $\mathrm{x}$ - and $\mathrm{y}$-coordinates with a 846 resolution of $0.205 \mathrm{~cm} /$ pixel recorded by the system without further binning. Encoding was performed 847 with a random forest encoder using 100 trees and 5-fold cross-validation with randomized folds (Benjamin 848 et al., 2018). Encoding over time was performed using continuous folds to preserve the order in time. 849 Encoding quality was measured with the Poisson pseudo- $\mathrm{R}^{2}$ score and averaged over folds. Statistical 850 comparisons of encoding quality for individual predictors, and comparisons of improvement in encoding 851 quality above all other predictors, were made using Wilcoxon's signed-rank test and used the average of 
852 the $\mathrm{A} 1$ and V2L (improvement in) encoding quality for each predictor. Reported confidence bounds 853 correspond to 95\% bootstrap confidence intervals, computed with the Seaborn Python library (v0.9.0, 854 2018).

\section{Decoding of animal position}

857 The position of the rat was decoded from the neuronal data recorded from A1 or V2L if a session included 858 at least 16 neurons from that area showing a rate map peak $>2 \mathrm{~Hz}$; only those neurons were included for 859 each session. This number was determined to provide a balance between decoding quality and number 860 of included sessions. Spikes were binned in $\mathbf{4 0 0} \mathrm{ms}$ bins, and the true position (i.e. the actual position of 861 the rat on the track) at every timeframe was assigned to a spatial bin on the linearized track (total of 35 862 bins, bin size $\sim 9.8 \mathrm{~cm}$ ). When the linear position changed spatial bins within a temporal bin, the position 863 was assigned to the spatial bin which occurred most often within the temporal bin. Running speed was 864 linearly interpolated at the centers of the temporal bins. Samples with speed $<0.1 \mathrm{~m} / \mathrm{s}$ and with spike 865 count $<5$ were excluded. A Bayesian classifier was employed to predict the spatial bin occupied by the rat 866 on the basis of the temporally binned neural data (Davidson et al., 2009). A 5-fold cross-validation routine 867 with shuffling was used, with identical shuffling (i.e. similar sized training set for each fold) across the two 868 areas for a given session.

869 Decoding errors were used as a main metric for decoding performance and computed as the 870 Euclidian distance between the centers of the true and the decoded spatial bin in 2D space. Pearson 871 correlations of instantaneous decoding errors between the two cortical areas in time were calculated to 872 assess whether A1 and V2L encoded the same position. This was performed separately for the error in the $873 \mathrm{x}$-direction and that in the $\mathrm{y}$-direction in 2D space, to preserve the directionality of the error in addition 874 to its magnitude.

875 Instantaneous error correlations resulting from these computations were compared with error 876 correlations computed following shuffling of the errors within the same spatial bin and running speed 877 range (Saleem et al., 2018). Running speed bins were defined per session by taking the full range of speeds 878 and subdividing it into 5 equipopulated bins. Significance of differences in error correlations before and 879 after shuffling were tested using Wilcoxon's signed-rank tests. Joint error density maps for A1 and V2L 880 were computed for the error correlations of recorded and shuffled data and for the $x$ - and $y$-direction 881 separately. Joint error density maps were averaged across all included sessions and smoothed with a 882 Gaussian filter with a standard deviation of 4 spatial bins. The relative probability of observing an error of 
a particular size and direction in the recorded versus shuffled data was calculated by taking the difference between the actual and shuffled joint density maps and dividing by the shuffled map. on the size of the population. First, for each included session, 50 unique, random groups of units were selected for each ensemble size (ranging from 5 to the maximum number of units in each session minus one). Then, the decoding analysis was performed for each group before averaging decoding performance across the groups. For the largest ensembles, with one fewer unit than the session total, it was not possible to create 50 unique groups. For these ensembles some groups were included twice. Crossvalidation was performed for every group by splitting the data into a training set (80\%) and test set (20\%), with identical shuffling across all groups of a session.

To exclude the possibility that the observed correlations in instantaneous decoding errors are a result of decoding artifacts in sessions with poor decoding, Pearson's correlation was computed for the average decoding errors and the correlation in instantaneous decoding errors across sessions, for A1 and V2L average errors separately and for instantaneous errors in the $\mathrm{X}$ - and Y-directions separately.

\section{Seaborn Python reference (no journal article associated)}

\section{Conflict of interest statement}

902 The authors declare no financial or non-financial competing interests.

905 This study was financially supported by Netherlands Organization for Scientific Research VENI Grant 906863.11 .010 to C.S.L. and by the European Union's Horizon 2020 Framework Program for Research and 907 Innovation under the Specific Grant Agreement No. 945539 (Human Brain Project SGA3). We thank 908 Kenneth D. Harris and A. David Redish for the availability of unit isolation software KlustaKwik and MClust, 909 respectively. We also gratefully acknowledge the use of a Seaborn Python library in our study (v0.9.0, DOI 910 10.5281/zenodo.1313201, 2018). The work of the Technology Center at the University of Amsterdam for 911 building the recording setup and the tetrode microdrives is highly appreciated. 
A
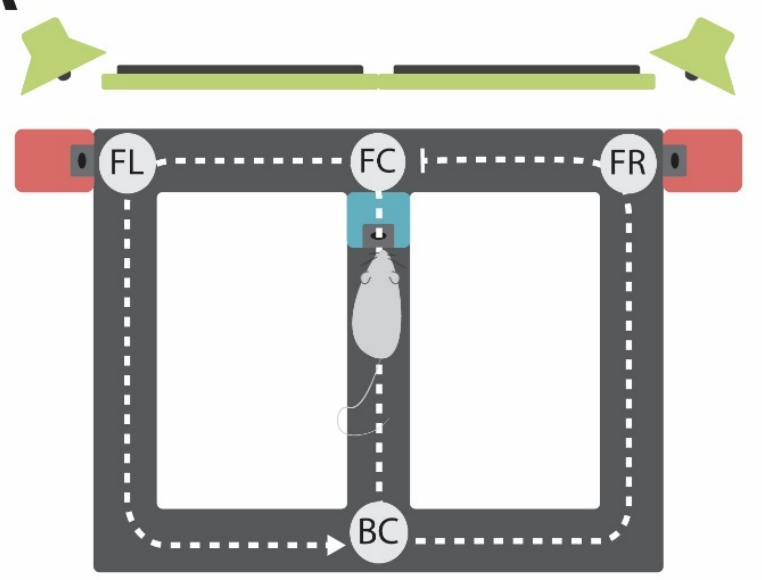

B

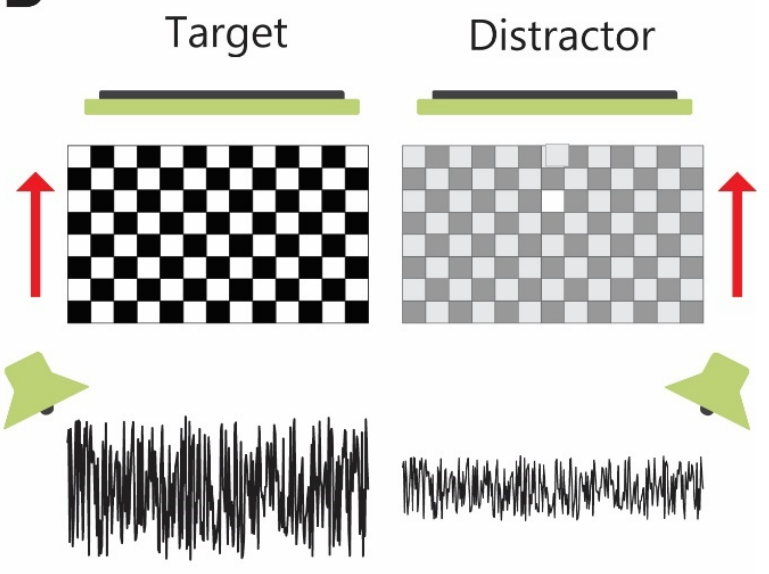

C NP center

Stimulus

NP/Lick

Reward

A1 Unit

V2L Unit

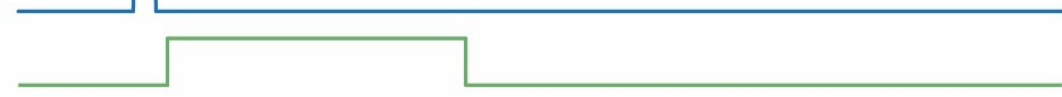

Movement

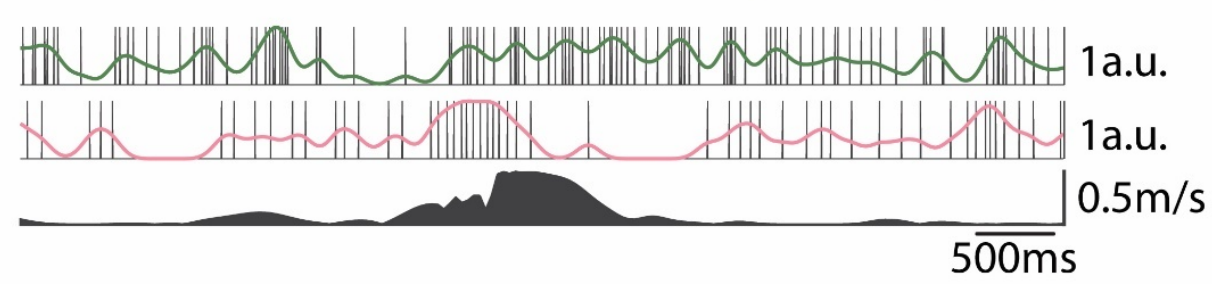

915 Figure 1: Behavioral apparatus and task. (A) Rats performed a discrimination task on an automatized figure-8 track, which was located in a dark, sound-attenuated laboratory room without other salient visual cues. At the front center T-junction (FC), rats responded to audio-visual stimuli presented from 2 screens and 2 speakers located in front of the track (green) by running to the track's side corresponding to where the most salient stimulus was presented. Rats were rewarded for a correct response with sucrose solution at the ports to the sides of the front alley (pink squares). The dotted line indicates how the track was linearized for analysis. FL: front left, FR: front right, BC: back center. (B) Example of a set of stimuli. Stimuli

922 were visual (moving checkerboard), auditory (filtered white noise) or audiovisual. In multisensory trials,

923 target auditory and visual stimuli were always presented at the same side of the track. The rat had to 924 respond to the most salient stimulus (highest contrast and/or volume) and discard the distractor stimulus 925 (which was less salient). (C) Trial layout and example A1 and V2L spike trains during seven seconds of a leftward trial with a correct response. NP center: blue line indicates the timing of the nose poke (NP) in 
927 the central well to initiate stimulus onset. Stimulus: the time interval during which the stimulus was

928 presented (2s; green line). NP/Lick: the time of the rat's nose poke into the left reward well is indicated

929 by the red shaded area and the individual licks by the vertical tick marks. A1 / V2L Units: Single unit spikes

930 are indicated by the black vertical tick marks, whereas solid lines indicate Gaussian-smoothed spike trains.

931 Firing rates are observed to fluctuate in relation to stimuli and locations across the maze. Movement:

932 speed of the rat along the linear trajectory.

933 

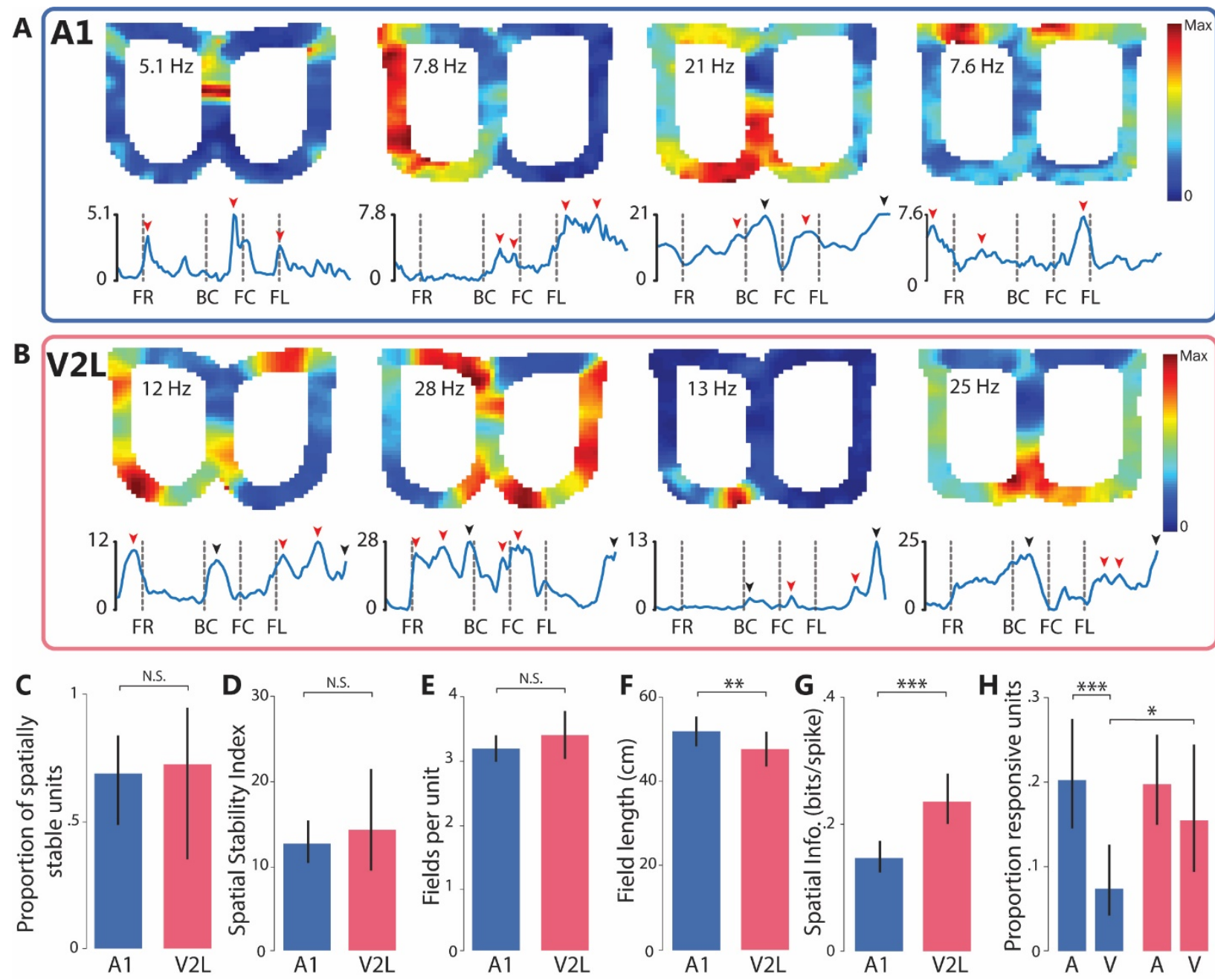

Figure 2: Spatially localized firing patterns of A1 and V2L units. (A) Top panels. Rate maps indicating the spatial firing-rate distribution of four example A1 units. The firing rate of the units is color-coded and the peak rates are indicated in the left wing of the map. Bottom panels. Firing rate distributions along the linearized version of the track corresponding to the rate maps shown in the top panels. Red arrow heads indicate individual firing field peaks. Black arrow heads indicate a single firing field peak that spans across an edge of the linearized track. Abbreviations below linear rate maps refer to landmark locations (fig. 1A).

942 (B) as (A) but for four example V2L units. (C) Estimate of the proportion of spatially stable units in the A1 943 and V2L populations. Black vertical lines throughout (C-H) indicate 95\% confidence intervals (N.S.: $p>$ 9440.05, F-test). (D) Spatial stability index of spatially stable units. (E) Number of firing fields per spatially stable unit (F) Field length per unit $(* *, p<0.01)$. (G) Spatial information of spatially stable units $(* * *, p<$ 
947 visual stimuli $(*, p<0.05)$.

948

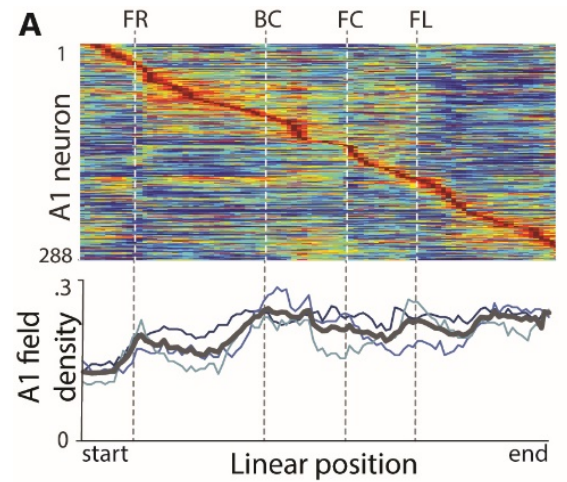

D

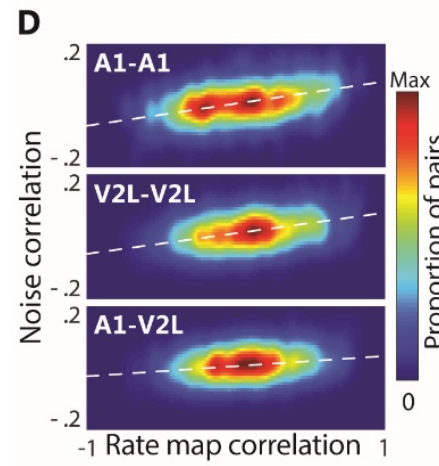

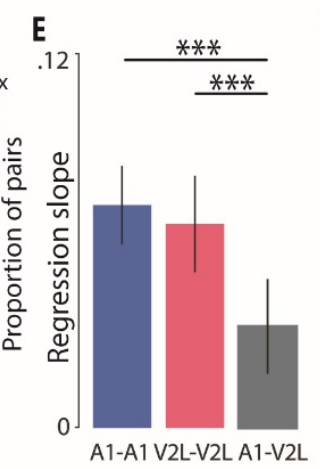

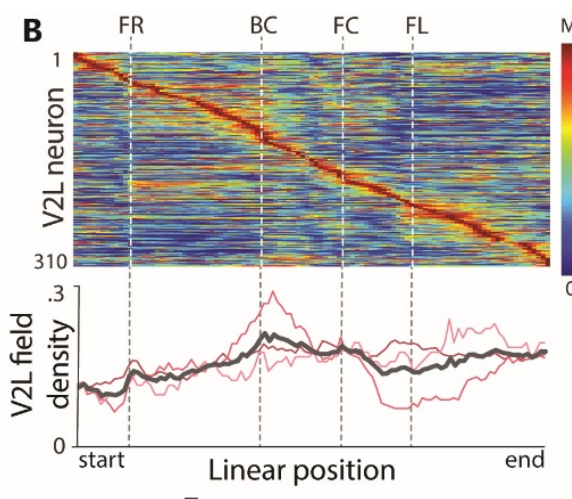

F
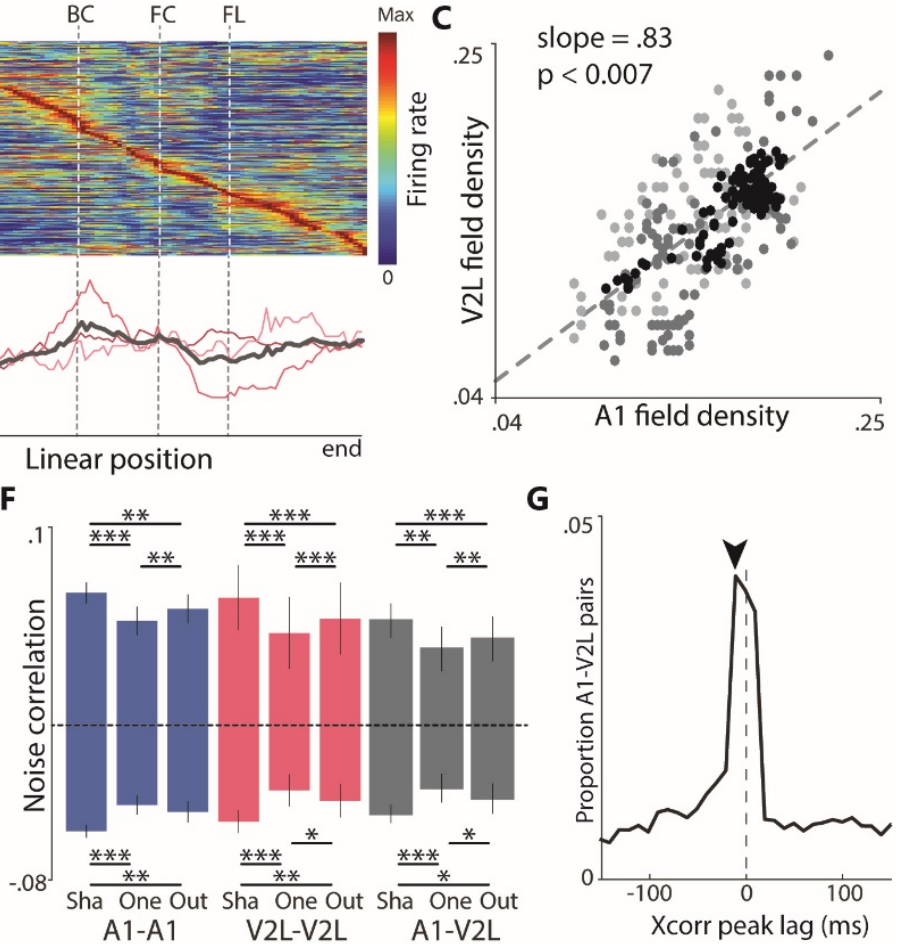

950

Figure 3: Spatial and temporal firing relations between A1 and V2L neurons. (A) Top panel. Joint linear rate map including all individual, spatially stable units recorded from A1. Firing rate is color-coded and the individual rate maps are sorted by peak location. Bottom panel. The firing field density across the spatial bins on the track are shown for the individual rats (thin, colored lines) and the mean across rats (thick, grey lines). The firing field density expresses for each spatial bin the proportion of individual firing fields that include that bin. (B) as (A) but for V2L. (C) Correlation between firing field densities of A1 and V2L across spatial bins. Dots correspond to individual spatial bins; different shades of grey correspond to individual rats. The dashed line indicates the linear regression of mean field densities across rats according to the linear mixed effects model. The value of $p$ indicates the significance of the regression line. (D) Heat maps show the proportions of cell pairs averaged across rats as a function of observed rate map correlations and noise correlations for A1-A1 pairs (top), V2L-V2L pairs (middle) and A1-V2L pairs (bottom). White, dashed lines indicate regression lines from a linear mixed effects model, indicating the significant, positive relationship between rate map correlations and noise correlations. (E) Bars indicate 
964 the slope of the regression of noise correlations on rate map correlations (white, dashed lines in D) for

965 the different single-unit pair types, with error bars indicating 95\% confidence bounds versus 0 . Asterisks

966 indicate a significant difference between groups (***: $p<0.001)$. (F) Mean pairwise noise correlations for

967 the within and between-area pairs and field configuration presented for significantly positively correlated

968 pairs and significantly negatively correlated pairs separately. Sha: locations shared between firing fields

969 of both units; One: locations exclusively appearing in the firing field of one of the two units, but not the

970 other; Out: locations outside of the firing fields of either unit. Error bars are $95 \%$ confidence bounds versus

9710 . Asterisks indicate a significant difference between groups (***:p<0.001, **:p<0.01, *: p<0.05). (G)

972 Proportions of A1-V2L unit pairs with observed temporal cross-correlation peak lags, averaged across rats.

973 Black arrow indicates the lag with the highest average proportion of pairs. Negative lags indicate that

974 activity of the $\mathrm{A} 1$ unit precedes that of the V2L unit. 


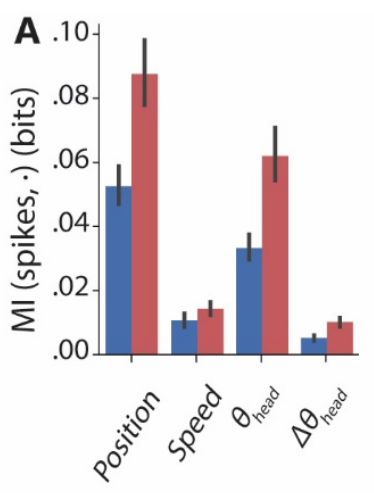

D
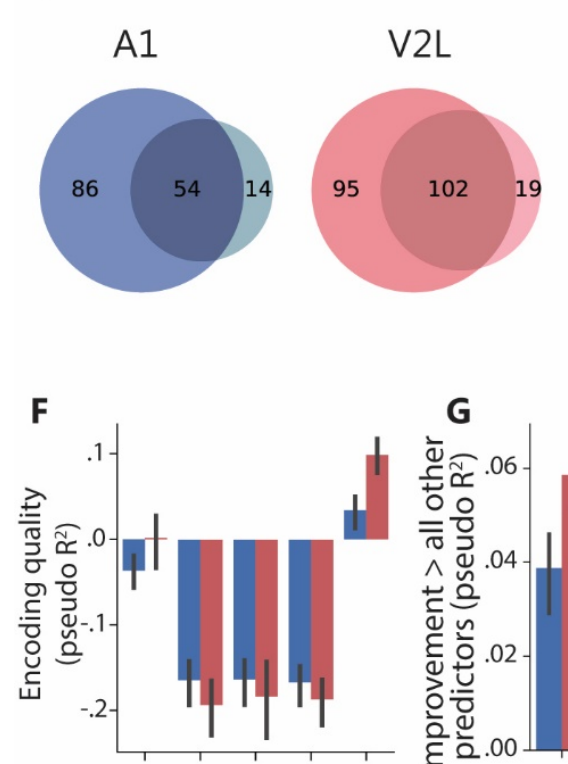

6

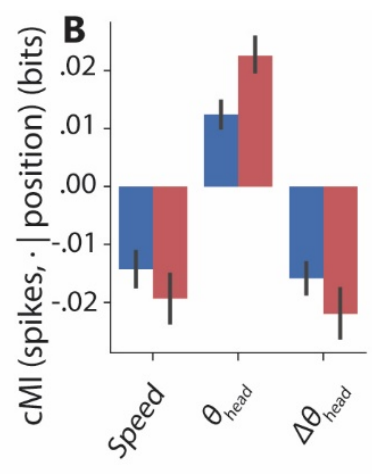

E
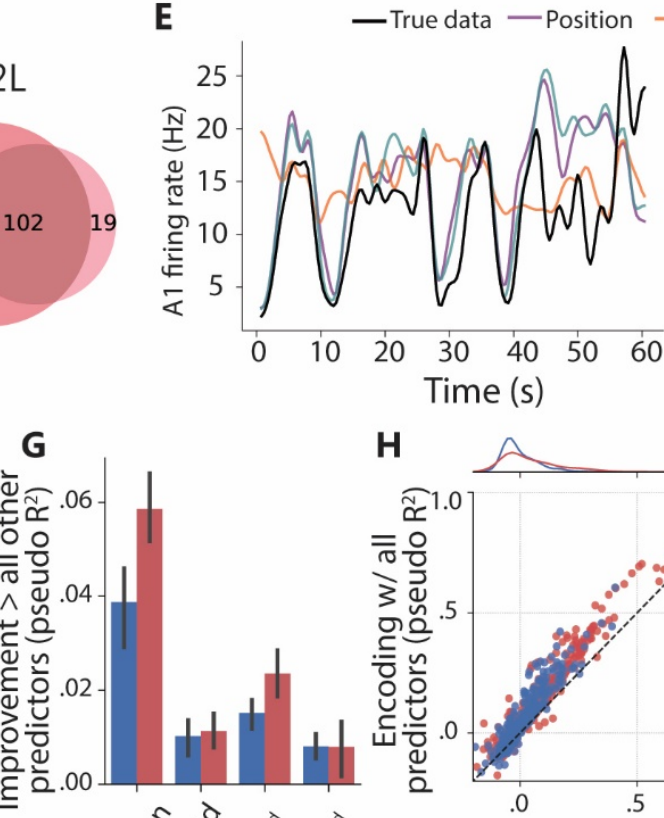

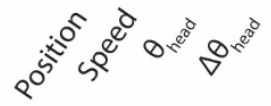

H

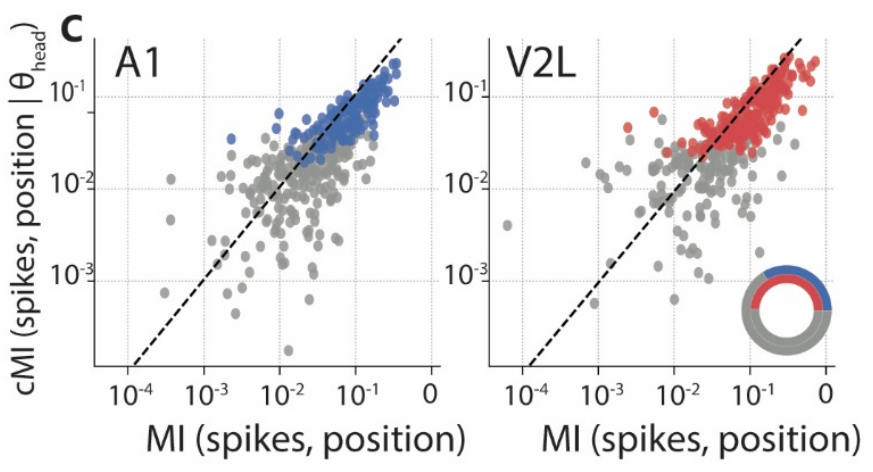

-True data - Position - $\theta_{\text {head }}$ - All predictors
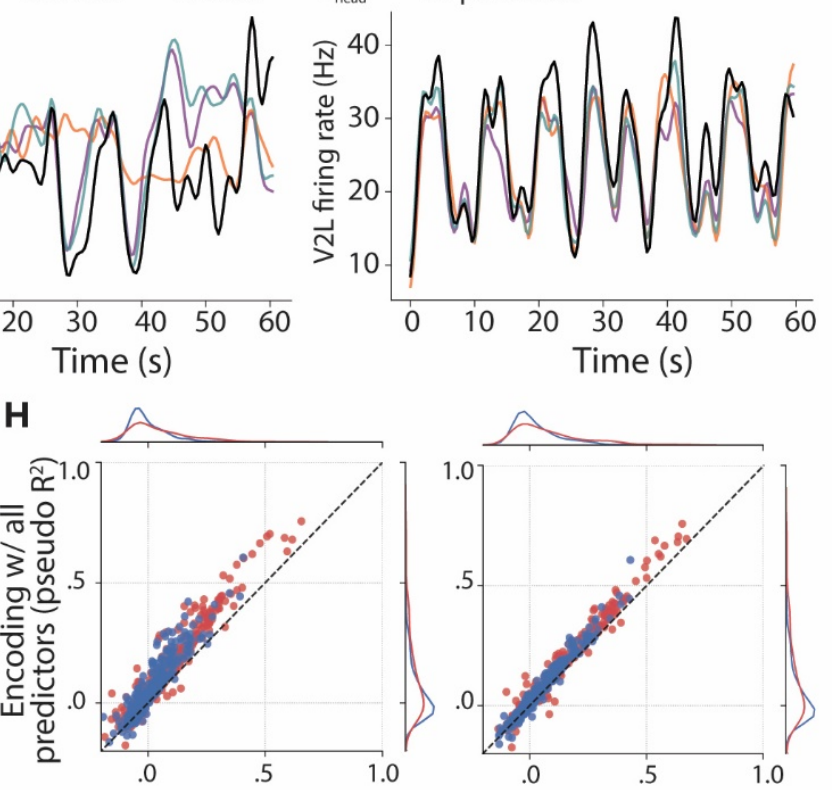

Encoding w/ all predictors Encoding w/ all predictors exc. position (pseudo $\mathrm{R}^{2}$ )

Figure 4: Encoding: predicting single-unit spiking activity from behavioral factors. (A) Average mutual information (MI) between spiking activity of $\mathrm{A} 1$ (blue) and $\mathrm{V} 2 \mathrm{~L}$ (red) single neurons and the behavioral factors position, running speed, head direction $\left(\theta_{\text {head }}\right)$, and head direction change $\left(\Delta \theta_{\text {head }}\right)$. Error bars represent $95 \%$ bootstrapped confidence bounds. (B) Debiased, conditional mutual information (cMI) between spiking activity and behavioral factors speed, head direction and changes in head direction, conditional on position. Error bars as in A. (C) Relationship between the MI of spikes and position and the CMI between spikes and position conditional on head direction for all spatially stable single units for A1 (left) and V2L (right). Blue/red points mark units with significant cMl about position conditional on $\theta_{\text {head, }}$ indicating that these units carry significant information on position that cannot be explained by $\theta_{\text {head. }}$. Inset shows in color the number of units per area showing significant $\mathrm{cMI}$ about position conditional on $\theta_{\text {head }}$ as 
987 fraction of the total number of units (grey). (D) Venn diagrams showing for each area the number of

988 neurons transmitting significant cMI(spikes, position $\left.\mid \theta_{\text {head }}\right)$ (dark color) and cMI(spikes, $\theta_{\text {head }} \mid$ position)

989 (light color). Overlapping region indicates neurons which transmit significant information on both position

990 and $\theta_{\text {head, }}$ neither of which can be explained entirely by the other factor. (E) A random forest encoder was

991 used to predict spiking behavior on the basis of position, running speed, $\theta_{\text {head }}$ and $\Delta \theta_{\text {head. }}$. Figure shows 60

992 s of firing rate of an example A1 unit (black line; left panel) and V2L unit (black line; right panel) and the

993 predicted firing rate based on the models including the different behavioral parameters (colored lines).

994 (F) Mean encoding quality across all A1 (blue) and V2L (red) units using single behavioral factors as

995 predictor and using all predictors. Error bars as in A. (G) Mean improvement in encoding quality across all

996 single units following the addition of the indicated behavioral factor to a model already containing all

997 other factors. Error bars are 95\% confidence bounds. (H) The relationship between encoding quality of

998 individual single units when all predictors are considered and the encoding quality when all predictors

999 except head direction (left) or linear position (right) are considered. Points above the diagonal belong to

1000 units with improved encoding due to the inclusion of linear position/ head direction which cannot be

1001 attributed to any other included factor. Blue: A1 units, red: V2L units. Diagrams to the right and top of the

1002 main scatterplot show the empirical distributions of the data depicted in the scatterplots projected onto

1003 a single dimension.

1004 
A

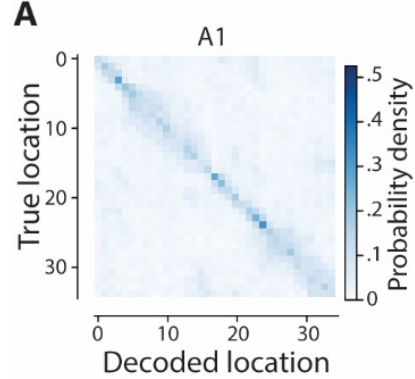

B

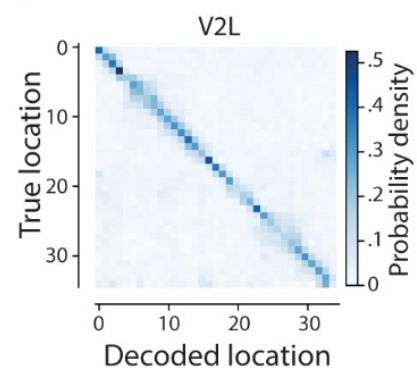

C

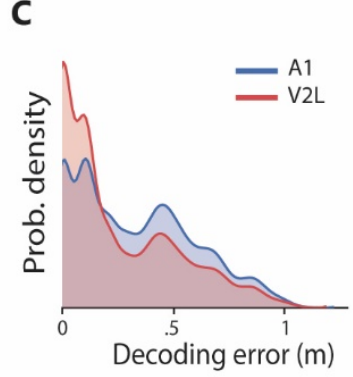

D

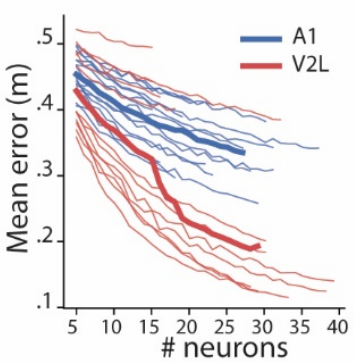

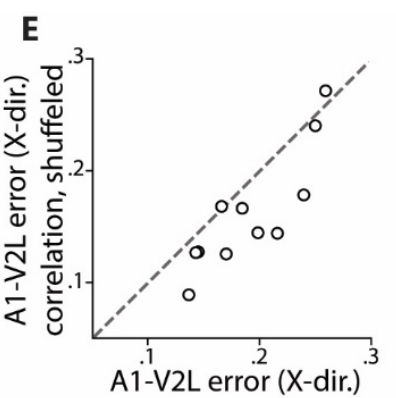

F correlation, measured

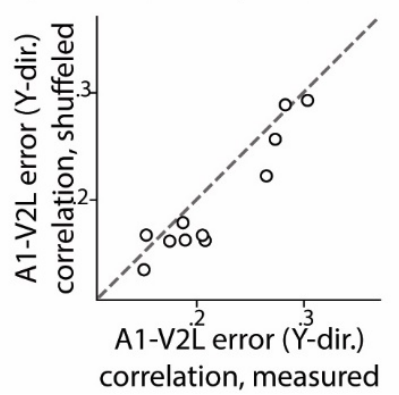

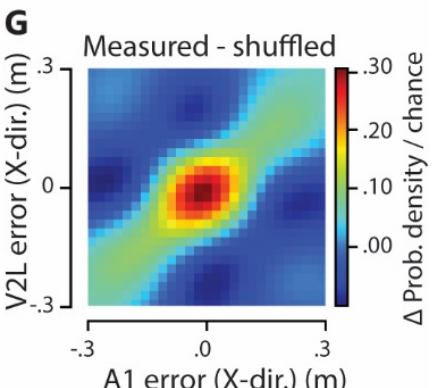

$\mathbf{H}$

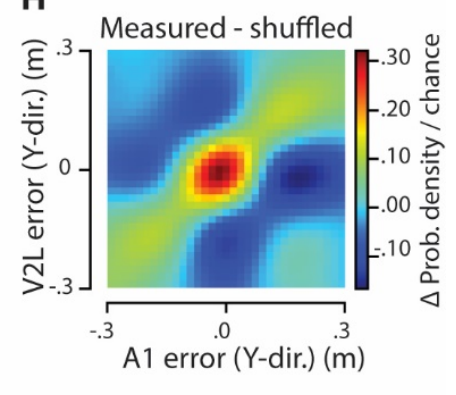

Figure 5: Spatial position can be reconstructed from A1 and V2L populations using a Bayesian decoder.

(A) Confusion matrices indicating the performance of Bayesian decoding of linearized position from A1 neuronal populations averaged across sessions. The probability that a sample of spiking activity is assigned by the decoder to the true location of the rat is coded in blue shades. (B) as (A) but for V2L populations. (C) Distribution of decoding errors (i.e. distances between the true and decoded position) across A1 (blue) and V2L (red) sessions. (D) Decoding error as a function of population size, obtained by randomly selecting units from the neuronal populations. Thin lines indicate means for individual sessions for A1 (blue) and V2L (red). Thick lines indicate means over sessions. (E) Correlations of measured (abscissa) and shuffled 1017 (ordinate) instantaneous decoding errors in the X-dimension of the maze. Shuffling was performed inside 1018 the same spatial bin and within the same running speed range. (F) Same as (E) but for the Y-dimension. 1019 (G) The size and direction of instantaneous errors were correlated between A1 and V2L. Color coded is 1020 the frequency of observing an instantaneous decoding error of a particular size and direction in the X1021 dimension after subtracting the frequency of instantaneous errors of a particular size and direction following shuffling of the errors within the same spatial bin and speed range. E.g. a value of +0.3 indicates that an error of that particular size and direction is $30 \%$ more likely to be observed in actual data than in shuffled data. (H) Same as (G) but for the Y-dimension. 


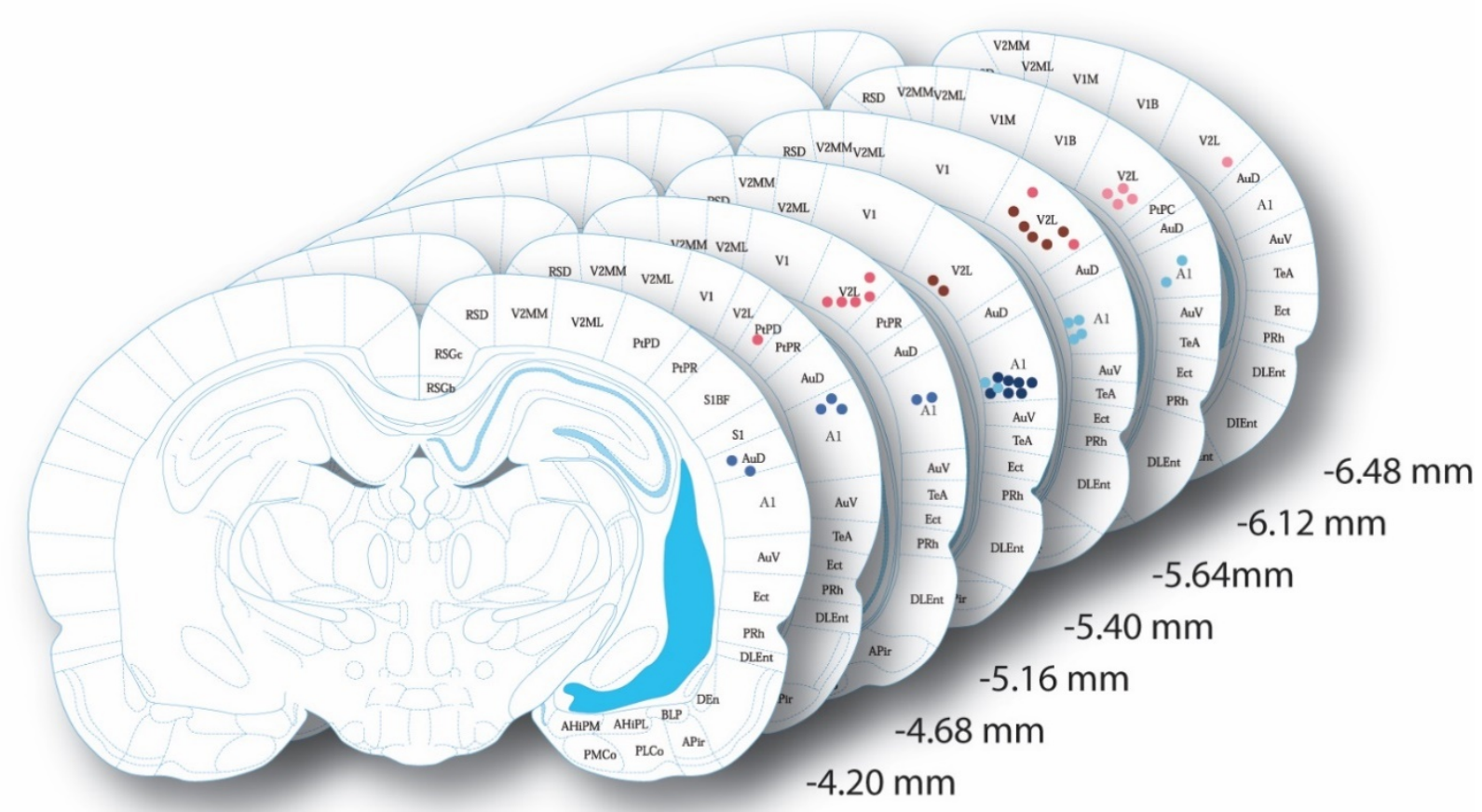

\section{Supplementary figure S1: Histology}

1029 Dots indicate locations of tetrode endpoints. Red dots represent endpoints of tetrodes targeted at V2L, 1030 blue dots endpoints of tetrodes targeted at A1. Three different shades were used to represent data from 1031 the three subjects. Light blue and light red correspond to the first subject, medium blue and medium red 1032 to the second subject and dark red and dark blue to the third subject. Numbers indicate distance from 1033 Bregma. Plates adapted from Paxinos \& Watson (2007). 
A

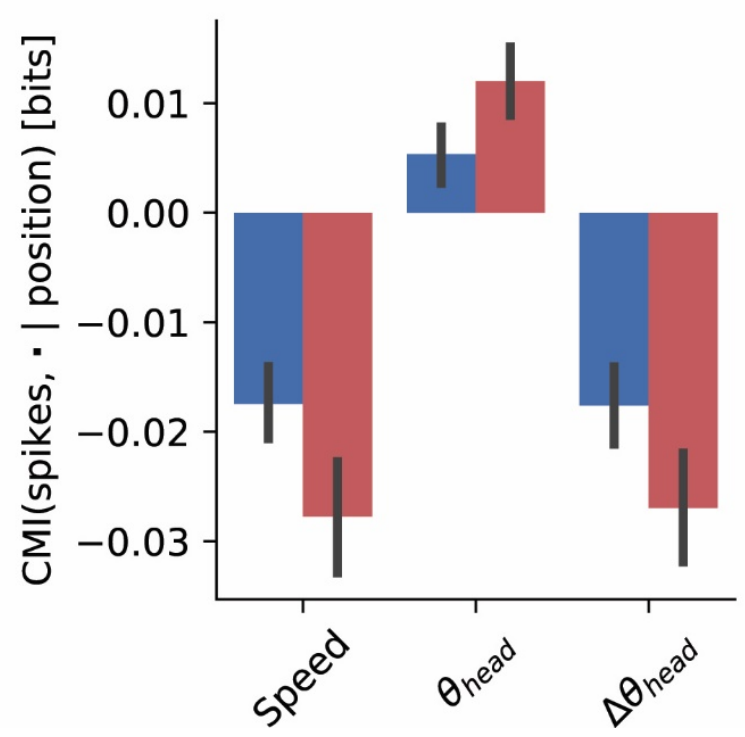

B

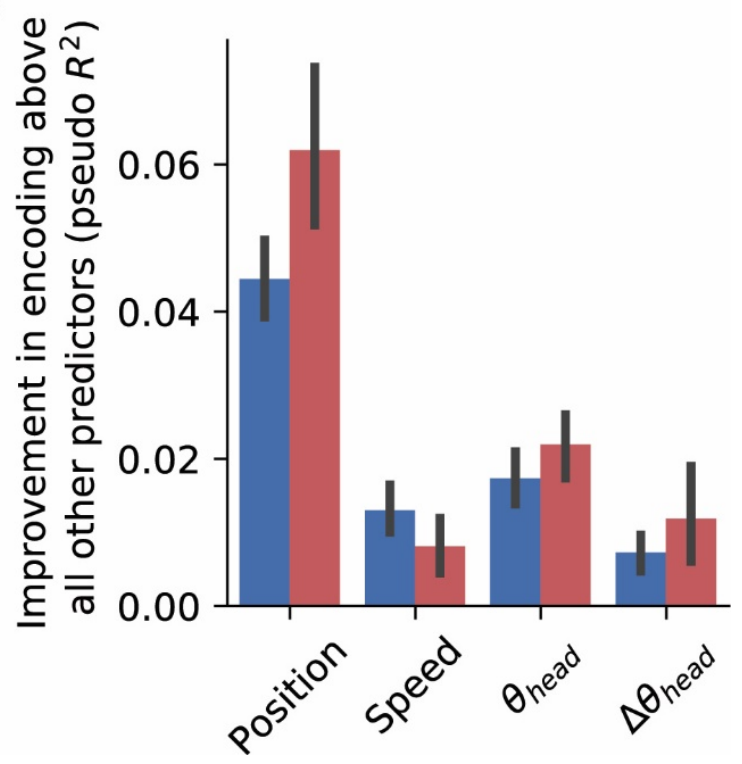

1038 (A) Debiased, conditional mutual information (cMI) between spiking activity and behavioral factors speed, 1039 head direction and changes in head direction, conditional on position (red bars: V2L; blue bars: A1). For 1040 this analysis, data from the period around stimulus presentation $\pm 1 \mathrm{~s}$ were ignored. Error bars represent $104195 \%$ bootstrapped confidence bounds. (B) Mean improvement in encoding quality of the random forest 1042 encoder across all single units following the addition of the indicated behavioral factor to a model already 1043 containing all other factors. For this analysis, data from the period around stimulus presentation $\pm 1 \mathrm{~s}$ 1044 were ignored. Error bars are 95\% confidence bounds. 

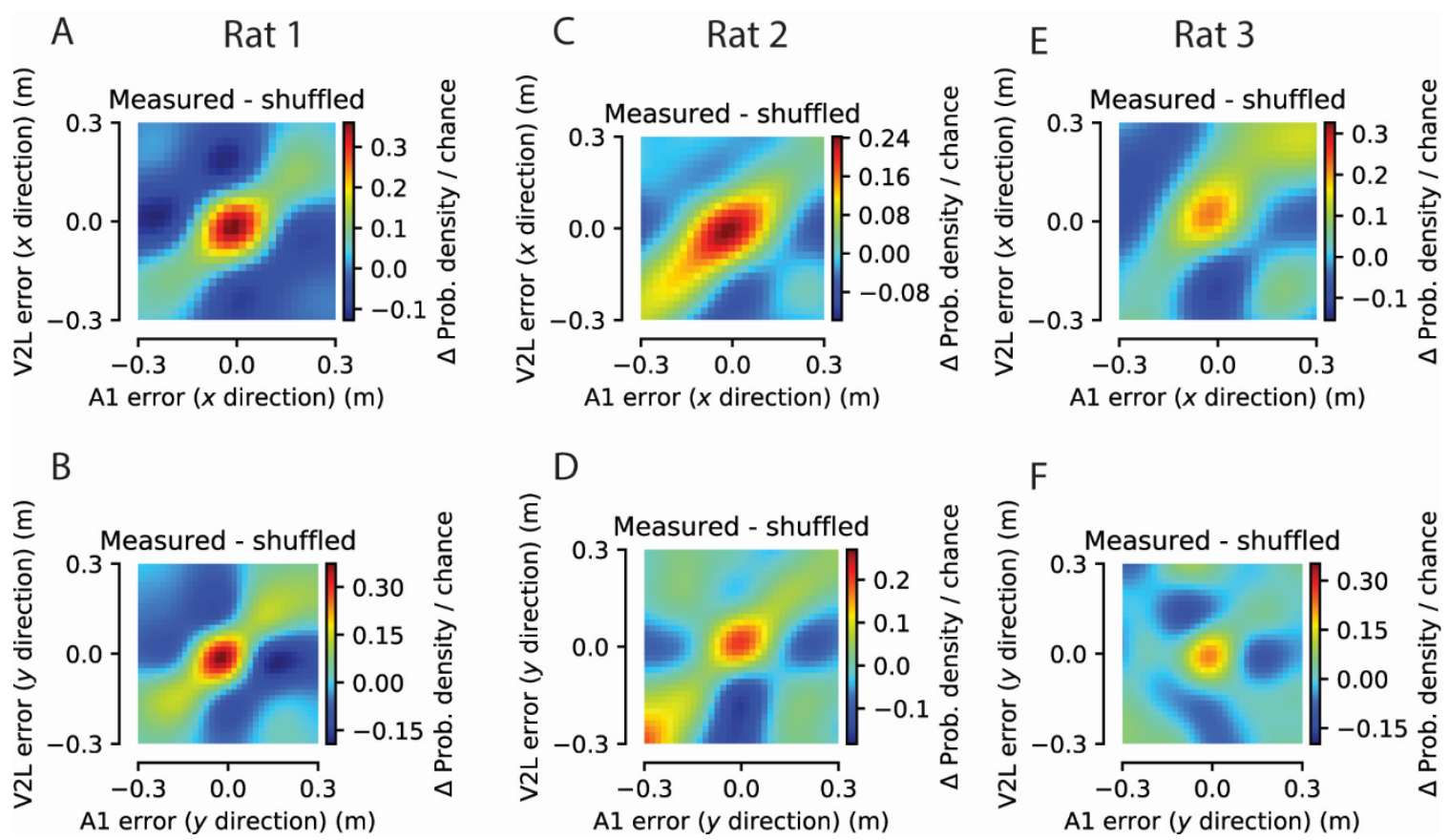

D

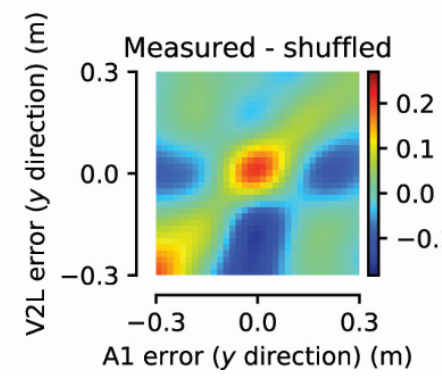

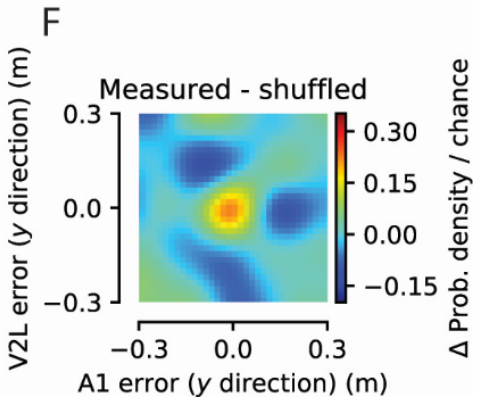

\section{Supplementary figure S3: Correlations in instantaneous decoding error per subject}

The size and direction of instantaneous decoding errors were correlated between $\mathrm{A} 1$ and V2L for individual subjects. (A) Color coded is the frequency of observing an instantaneous decoding error of a particular size and direction in the X-dimension (in A1, abscissa, vs. V2L, ordinate) relative to the frequency of instantaneous errors of a particular size and direction following shuffling of the errors within the same spatial bin and speed range. Data from the first subject. (B) as (A) but for the Y-dimension. (C) and (D), as (A) and (B) but for the second subject. (E) and (F) as (A) and (B) but for the third subject. 

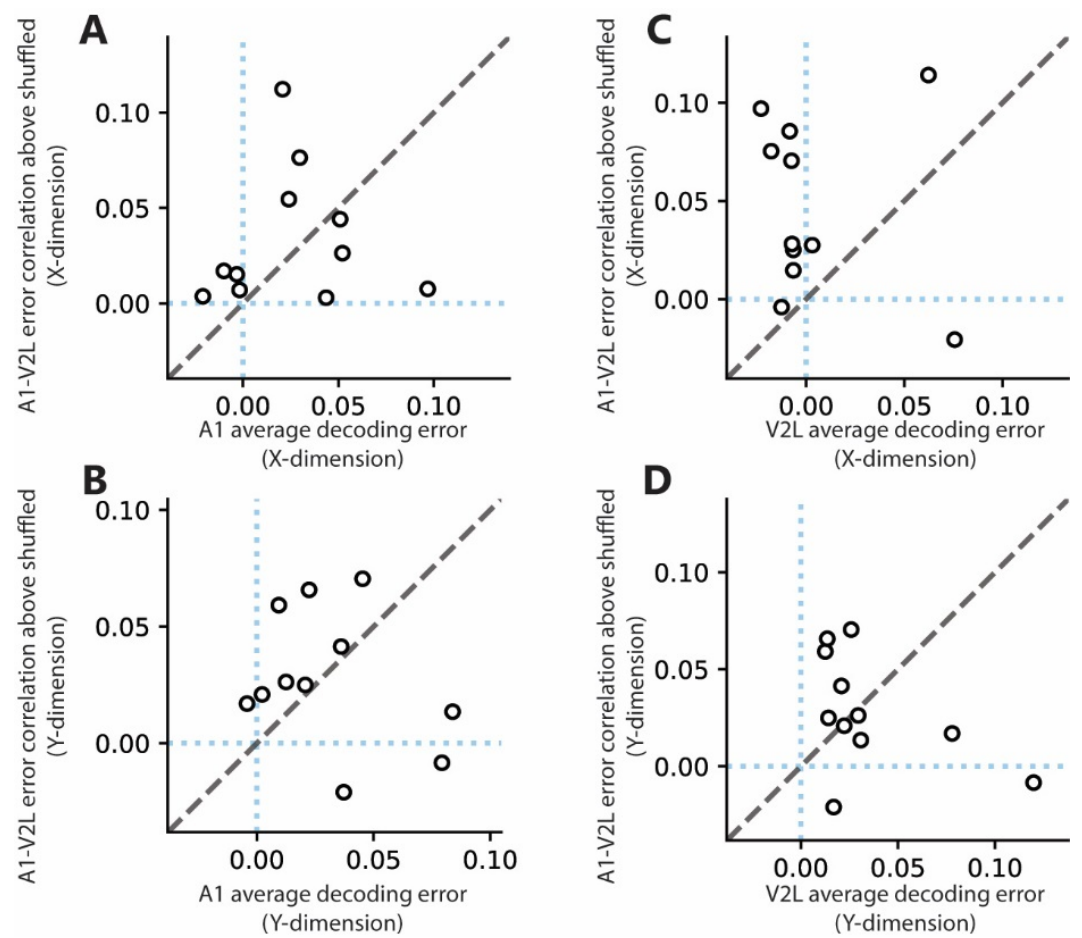

Supplementary figure S4: Average decoding error and instantaneous error correlations between areas are uncorrelated

(A) Using Pearson's correlation, we found no linear relationship between the average decoding error in the X-dimension in A1 and the average correlation in instantaneous decoding error in the X-dimension between $A 1$ and V2L. Each dot represents a session included in the decoding analysis for both sessions. (B) As (A) but for the Y-dimension. (C) As (A) but for the average decoding error in V2L. (D) as (C) but for 
1069

1070

1071

1072

1073

1074

1075

1076

1077

1078

1079

1080

1081

1082

1083

1084

1085

1086

1087

1088

1089

1090

1091

1092

1093

1094

1095

1096

1097

1098

1099

1100

1101

1102

1103

1104

1105

1106

1107

1108

1109

1110

1111

1112

1113

1114

1115

1116

1117

\section{References}

Averbeck, B.B., Latham, P.E., and Pouget, A. (2006). Neural correlations, population coding and computation. Nat Rev Neurosci 7, 358-366.

Ayaz, A., Saleem, Aman B., Schölvinck, Marieke L., and Carandini, M. (2013). Locomotion Controls Spatial Integration in Mouse Visual Cortex. Current Biology 23, 890-894.

Barresi, M., Grasso, C., Li Volsi, G., and Manzoni, D. (2013). Effects of body to head rotation on the labyrinthine responses of rat vestibular neurons. Neuroscience 244, 134-146.

Benjamin, A.S., Fernandes, H.L., Tomlinson, T., Ramkumar, P., VerSteeg, C., Chowdhury, R.H., Miller, L.E., and Kording, K.P. (2018). Modern Machine Learning as a Benchmark for Fitting Neural Responses. Frontiers in Computational Neuroscience 12.

Bizley, J.K., Nodal, F.R., Bajo, V.M., Nelken, I., and King, A.J. (2007). Physiological and Anatomical Evidence for Multisensory Interactions in Auditory Cortex. Cerebral Cortex 17, 21722189.

Bos, J.J., Vinck, M., Marchesi, P., Keestra, A., van Mourik-Donga, L.A., Jackson, J.C., Verschure, P.F.M.J., and Pennartz, C.M.A. (2019). Multiplexing of Information about Self and Others in Hippocampal Ensembles. Cell Reports 29, 3859-3871.e3856.

Bos, J.J., Vinck, M., van Mourik-Donga, L.A., Jackson, J.C., Witter, M.P., and Pennartz, C.M.A. (2017). Perirhinal firing patterns are sustained across large spatial segments of the task environment. Nature Communications 8, 15602.

Brosch, M., Selezneva, E., and Scheich, H. (2005). Nonauditory Events of a Behavioral Procedure Activate Auditory Cortex of Highly Trained Monkeys. Journal of Neuroscience 25, 6797-6806.

Budinger, E., and Scheich, H. (2009). Anatomical connections suitable for the direct processing of neuronal information of different modalities via the rodent primary auditory cortex. Hearing Research 258, 16-27.

Campi, K.L., Bales, K.L., Grunewald, R., and Krubitzer, L. (2010). Connections of Auditory and Visual Cortex in the Prairie Vole (Microtus ochrogaster): Evidence for Multisensory Processing in Primary Sensory Areas. Cerebral Cortex 20, 89-108.

Chen, X., DeAngelis, G.C., and Angelaki, D.E. (2018). Flexible egocentric and allocentric representations of heading signals in parietal cortex. Proceedings of the National Academy of Sciences of the United States of America 115, E3305-E3312.

Clark, B.J., Simmons, C.M., Berkowitz, L.E., and Wilber, A.A. (2018). The retrosplenial-parietal network and reference frame coordination for spatial navigation. Behavioral Neuroscience 132, 416-429.

Clemens, L.E., Jansson, E.K.H., Portal, E., Riess, O., and Nguyen, H.P. (2014). A behavioral comparison of the common laboratory rat strains Lister Hooded, Lewis, Fischer 344 and Wistar in an automated homecage system. Genes, Brain, and Behavior 13, 305-321.

Cullen, K.E. (2014). The neural encoding of self-generated and externally applied movement: implications for the perception of self-motion and spatial memory. Frontiers in Integrative Neuroscience 7, 108.

D'Souza, R.D., Meier, A.M., Bista, P., Wang, Q., and Burkhalter, A. (2016). Recruitment of inhibition and excitation across mouse visual cortex depends on the hierarchy of interconnecting areas. eLife 5, e19332.

Davidson, T.J., Kloosterman, F., and Wilson, M.A. (2009). Hippocampal replay of extended experience. Neuron 63, 497-507.

DiCarlo, J.J., and Cox, D.D. (2007). Untangling invariant object recognition. Trends in Cognitive Sciences 11, 333-341.

Ecker, A.S., Berens, P., Keliris, G.A., Bethge, M., Logothetis, N.K., and Tolias, A.S. (2010). Decorrelated neuronal firing in cortical microcircuits. Science 327, 584-587. 
Eichenbaum, H. (2000). A cortical-hippocampal system for declarative memory. Nat Rev Neurosci 1, 41-50.

Felleman, D.J., and Van Essen, D.C. (1991). Distributed hierarchical processing in the primate cerebral cortex. Cerebral Cortex (New York, NY: 1991) 1, 1-47. sensory cue integration and the physiology of multisensory neurons. Nat Rev Neurosci 14, 429442. Experience-dependent spatial expectations in mouse visual cortex. Nature Neuroscience 19, 1658-1664.

Fournier, J., Saleem, A.B., Diamanti, E.M., Wells, M.J., Harris, K.D., and Carandini, M. (2019). Modulation of visual cortex by hippocampal signals. bioRxiv, 586917 . Biology 30, 3811-3817.e3816. (2016). Structures of Neural Correlation and How They Favor Coding. Neuron 89, 409-422. Friston, K. (2005). A theory of cortical responses. Philosophical Transactions of the Royal Society of London Series B, Biological Sciences 360, 815-836. parahippocampal region in the rat: the perirhinal and postrhinal cortices. Hippocampus 17, 709722. and Burkhalter, A. (2018). The Mouse Cortical Connectome, Characterized by an Ultra-Dense Cortical Graph, Maintains Specificity by Distinct Connectivity Profiles. Neuron 97, 698-715.e610. Gavornik, J.P., and Bear, M.F. (2014). Learned spatiotemporal sequence recognition and prediction in primary visual cortex. Nature Neuroscience 17, 732-737.

Goltstein, P.M., Coffey, E.B.J., Roelfsema, P.R., and Pennartz, C.M.A. (2013). In Vivo TwoPhoton Ca2+ Imaging Reveals Selective Reward Effects on Stimulus-Specific Assemblies in Mouse Visual Cortex. Journal of Neuroscience 33, 11540-11555.

Goltstein, P.M., Montijn, J.S., and Pennartz, C.M.A. (2015). Effects of Isoflurane Anesthesia on Ensemble Patterns of Ca2+ Activity in Mouse V1: Reduced Direction Selectivity Independent of Increased Correlations in Cellular Activity. PLOS ONE 10, e0118277.

Gray, C.M., Maldonado, P.E., Wilson, M., and McNaughton, B. (1995). Tetrodes markedly improve the reliability and yield of multiple single-unit isolation from multi-unit recordings in cat striate cortex. Journal of neuroscience methods 63, 43-54.

Groenewegen, H.J., and Uylings, H.B. (2000). The prefrontal cortex and the integration of sensory, limbic and autonomic information. Prog Brain Res 126, 3-28.

Guitchounts, G., Masís, J., Wolff, S.B.E., and Cox, D. (2020). Encoding of 3D Head Orienting Movements in the Primary Visual Cortex. Neuron 108, 512-525.e514.

Haggerty, D.C., and Ji, D. (2015). Activities of visual cortical and hippocampal neurons cofluctuate in freely moving rats during spatial behavior. eLife 4, e08902.

Hansen, B.J., Chelaru, M.I., and Dragoi, V. (2012). Correlated Variability in Laminar Cortical Circuits. Neuron 76, 590-602.

Harris, J.A., Mihalas, S., Hirokawa, K.E., Whitesell, J.D., Choi, H., Bernard, A., Bohn, P., Caldejon, S., Casal, L., Cho, A., et al. (2019). Hierarchical organization of cortical and thalamic connectivity. Nature 575, 195-202.

Hawkins, J., Ahmad, S., and Cui, Y. (2017). A Theory of How Columns in the Neocortex Enable Learning the Structure of the World. Frontiers in neural circuits 11, 81.

1168 sounds. Journal of Neurophysiology 41, 963-976. 
1169

1170

1171

1172

1173

1174

1175

1176

1177

1178

1179

1180

1181

1182

1183

1184

1185

1186

1187

1188

1189

1190

1191

1192

1193

1194

1195

1196

1197

1198

1199

1200

1201

1202

1203

1204

1205

1206

1207

1208

1209

1210

1211

1212

1213

1214

1215

1216

1217

1218

1219

Hubel, D.H., and Wiesel, T.N. (1962). Receptive fields, binocular interaction and functional architecture in the cat's visual cortex. The Journal of physiology 160, 106-154.

Ibrahim, L.A., Mesik, L., Ji, X.-y., Fang, Q., Li, H.-f., Li, Y.-t., Zingg, B., Zhang, L.I., and Tao, H.W. (2016). Cross-Modality Sharpening of Visual Cortical Processing through Layer-1-Mediated Inhibition and Disinhibition. Neuron 89, 1031-1045.

lurilli, G., Ghezzi, D., Olcese, U., Lassi, G., Nazzaro, C., Tonini, R., Tucci, V., Benfenati, F., and Medini, P. (2012). Sound-driven synaptic inhibition in primary visual cortex. Neuron 73, 814-828. Jaramillo, S., and Zador, A.M. (2011). The auditory cortex mediates the perceptual effects of acoustic temporal expectation. Nature Neuroscience 14, 246-251.

Jenkins, W.M., and Merzenich, M.M. (1984). Role of cat primary auditory cortex for soundlocalization behavior. Journal of Neurophysiology 52, 819-847.

Ji, D., and Wilson, M.A. (2007). Coordinated memory replay in the visual cortex and hippocampus during sleep. Nat Neurosci 10, 100-107.

Kadir, S.N., Goodman, D.F.M., and Harris, K.D. (2014). High-Dimensional Cluster Analysis with the Masked EM Algorithm. Neural Computation 26, 2379-2394.

Kavanagh, G.L., and Kelly, J.B. (1987). Contribution of auditory cortex to sound localization by the ferret (Mustela putorius). Journal of Neurophysiology 57, 1746-1766.

Keller, G.B., and Mrsic-Flogel, T.D. (2018). Predictive Processing: A Canonical Cortical Computation. Neuron 100, 424-435.

Knierim, J.J. (2002). Dynamic interactions between local surface cues, distal landmarks, and intrinsic circuitry in hippocampal place cells. Journal of Neuroscience 22, 6254-6264.

Knöpfel, T., Sweeney, Y., Radulescu, C.I., Zabouri, N., Doostdar, N., Clopath, C., and Barnes, S.J. (2019). Audio-visual experience strengthens multisensory assemblies in adult mouse visual cortex. Nature communications 10, 5684.

Krumin, M., Lee, J.J., Harris, K.D., and Carandini, M. (2018). Decision and navigation in mouse parietal cortex. eLife 7, e42583.

Lansink, C.S., Bakker, M., Buster, W., Lankelma, J., van der Blom, R., Westdorp, R., Joosten, R.N.J.M.A., McNaughton, B.L., and Pennartz, C.M.A. (2007). A split microdrive for simultaneous multi-electrode recordings from two brain areas in awake small animals. Journal of neuroscience methods $162,129-138$.

Lansink, C.S., Jackson, J.C., Lankelma, J.V., Ito, R., Robbins, T.W., Everitt, B.J., and Pennartz, C.M. (2012). Reward cues in space: commonalities and differences in neural coding by hippocampal and ventral striatal ensembles. J Neurosci 32, 12444-12459.

Laramée, M.E., Kurotani, T., Rockland, K.S., Bronchti, G., and Boire, D. (2011). Indirect pathway between the primary auditory and visual cortices through layer $\mathrm{V}$ pyramidal neurons in $\mathrm{V} 2 \mathrm{~L}$ in mouse and the effects of bilateral enucleation. European Journal of Neuroscience 34, 65-78.

Leinweber, M., Ward, D.R., Sobczak, J.M., Attinger, A., and Keller, G.B. (2017). A Sensorimotor Circuit in Mouse Cortex for Visual Flow Predictions. Neuron 95, 1420-1432.e1425.

Leutgeb, S., Leutgeb, J.K., Barnes, C.A., Moser, E.I., McNaughton, B.L., and Moser, M.B. (2005). Independent codes for spatial and episodic memory in hippocampal neuronal ensembles. Science 309, 619-623.

Lin, I.-C., Okun, M., Carandini, M., and Harris, K.D. (2015). The Nature of Shared Cortical Variability. Neuron 87, 644-656.

Lizier, J.T. (2014). JIDT: An Information-Theoretic Toolkit for Studying the Dynamics of Complex Systems. Frontiers in Robotics and AI 1.

Lopes, G., Bonacchi, N., Frazão, J., Neto, J.P., Atallah, B.V., Soares, S., Moreira, L., Matias, S., Itskov, P.M., Correia, P.A., et al. (2015). Bonsai: an event-based framework for processing and controlling data streams. Frontiers in Neuroinformatics 9.

Louie, K., and Wilson, M.A. (2001). Temporally structured replay of awake hippocampal ensemble activity during rapid eye movement sleep. Neuron 29, 145-156.

McGaugh, J.L. (2000). Memory--a century of consolidation. Science 287, 248-251. 

(1994). Cortical representation of motion during unrestrained spatial navigation in the rat. Cerebral Cortex (New York, NY: 1991) 4, 27-39.

Medrea, I., and Cullen, K.E. (2013). Multisensory integration in early vestibular processing in mice: the encoding of passive vs. active motion. Journal of Neurophysiology 110, 2704-2717. Meijer, G.T., Marchesi, P., Mejias, J.F., Montijn, J.S., Lansink, C.S., and Pennartz, C.M. (2020). Neural Correlates of Multisensory Detection Behavior: Comparison of Primary and Higher-Order Visual Cortex. Cell Reports 31, 107636.

Meijer, G.T., Mertens, P.E.C., Pennartz, C.M.A., Olcese, U., and Lansink, C.S. (2019). The circuit architecture of cortical multisensory processing: Distinct functions jointly operating within a common anatomical network. Prog Neurobiol 174, 1-15.

Meijer, G.T., Montijn, J.S., Pennartz, C.M.A., and Lansink, C.S. (2017). Audiovisual Modulation in Mouse Primary Visual Cortex Depends on Cross-Modal Stimulus Configuration and Congruency. The Journal of Neuroscience: The Official Journal of the Society for Neuroscience 37, 8783-8796.

Middlebrooks, J.C., and Pettigrew, J.D. (1981). Functional classes of neurons in primary auditory cortex of the cat distinguished by sensitivity to sound location. The Journal of Neuroscience: The Official Journal of the Society for Neuroscience 1, 107-120.

Miller, K.D. (2016). Canonical computations of cerebral cortex. Current Opinion in Neurobiology $37,75-84$.

Miller, M.W., and Vogt, B.A. (1984). Direct connections of rat visual cortex with sensory, motor, and association cortices. Journal of Comparative Neurology 226, 184-202.

Montijn, J.S., Meijer, G.T., Lansink, C.S., and Pennartz, C.M. (2016). Population-Level Neural Codes Are Robust to Single-Neuron Variability from a Multidimensional Coding Perspective. Cell Rep 16, 2486-2498.

Morrill, R.J., and Hasenstaub, A.R. (2018). Visual Information Present in Infragranular Layers of Mouse Auditory Cortex. J Neurosci 38, 2854-2862.

Namboodiri, Vijay Mohan K., Huertas, Marco A., Monk, Kevin J., Shouval, Harel Z., and Hussain Shuler, Marshall G. (2015). Visually Cued Action Timing in the Primary Visual Cortex. Neuron 86, 319-330.

Newby, F.D., DiGirolamo, M., Cotsonis, G.A., and Kutner, M.H. (1990). Model of spontaneous obesity in aging male Wistar rats. The American Journal of Physiology 259, R1117-1125.

Niell, C.M., and Stryker, M.P. (2010). Modulation of Visual Responses by Behavioral State in Mouse Visual Cortex. Neuron 65, 472-479.

Nitz, D.A. (2006). Tracking Route Progression in the Posterior Parietal Cortex. Neuron 49, 747756.

Niwa, M., Johnson, J.S., O'Connor, K.N., and Sutter, M.L. (2012). Activity Related to Perceptual Judgment and Action in Primary Auditory Cortex. Journal of Neuroscience 32, 3193-3210.

O'Keefe, J., and Nadel, L. (1978). The hippocampus as a cognitive map (Oxford: Clarendon Press).

Olcese, U., Bos, J.J., Vinck, M., Lankelma, J.V., Mourik-Donga, L.B.V., Schlumm, F., and Pennartz, C.M.A. (2016). Spike-Based Functional Connectivity in Cerebral Cortex and Hippocampus: Loss of Global Connectivity Is Coupled to Preservation of Local Connectivity During Non-REM Sleep. Journal of Neuroscience 36, 7676-7692.

Page, H.J.I., and Jeffery, K.J. (2018). Landmark-Based Updating of the Head Direction System by Retrosplenial Cortex: A Computational Model. Frontiers in Cellular Neuroscience 12, 191.

Pakan, J.M.P., Currie, S.P., Fischer, L., and Rochefort, N.L. (2018). The Impact of Visual Cues, Reward, and Motor Feedback on the Representation of Behaviorally Relevant Spatial Locations in Primary Visual Cortex. Cell Reports 24, 2521-2528.

Paxinos, G., and Watson, C. (2007). The Rat Brain in Stereotaxic Coordinates, 6th edn (Academic Press). 

Directed. Trends Cogn Sci 22, 137-153.

Pennartz, C.M.A., Dora, S., Muckli, L., and Lorteije, J.A.M. (2019). Towards a Unified View on Pathways and Functions of Neural Recurrent Processing. Trends in Neurosciences 42, 589-603. Picton, T.W., Hillyard, S.A., Krausz, H.I., and Galambos, R. (1974). Human auditory evoked potentials. I. Evaluation of components. Electroencephalogr Clin Neurophysiol 36, 179-190.

Rao, R.P.N., and Ballard, D.H. (1999). Predictive coding in the visual cortex: a functional interpretation of some extra-classical receptive-field effects. Nature Neuroscience 2, 79.

Rosenbaum, R., Smith, M.A., Kohn, A., Rubin, J.E., and Doiron, B. (2017). The spatial structure of correlated neuronal variability. Nature Neuroscience 20,107-114.

Rusu, S.I., and Pennartz, C.M.A. (2020). Learning, memory and consolidation mechanisms for behavioral control in hierarchically organized cortico-basal ganglia systems. Hippocampus 30 , 73-98.

Saleem, A.B., Diamanti, E.M., Fournier, J., Harris, K.D., and Carandini, M. (2018). Coherent encoding of subjective spatial position in visual cortex and hippocampus. Nature 562, 124. Sargolini, F., Fyhn, M., Hafting, T., McNaughton, B.L., Witter, M.P., Moser, M.-B., and Moser, E.I. (2006). Conjunctive representation of position, direction, and velocity in entorhinal cortex. Science (New York, NY) 312, 758-762. Scheich, H., Brechmann, A., Brosch, M., Budinger, E., and Ohl, F.W. (2007). The cognitive auditory cortex: Task-specificity of stimulus representations. Hearing Research 229, 213-224.

Schmitzer-Torbert, N., Jackson, J., Henze, D., Harris, K., and Redish, A.D. (2005). Quantitative measures of cluster quality for use in extracellular recordings. Neuroscience 131, 1-11.

Schulz, D.P.A., Sahani, M., and Carandini, M. (2015). Five key factors determining pairwise correlations in visual cortex. Journal of Neurophysiology.

Schürmann, T., Vogt, J., Christ, O., and Beckerle, P. (2019). The Bayesian causal inference model benefits from an informed prior to predict proprioceptive drift in the rubber foot illusion. Cogn Process 20, 447-457.

Shuler, M.G., and Bear, M.F. (2006). Reward Timing in the Primary Visual Cortex. Science 311, 1606-1609.

Skaggs, W.E., McNaughton, B.L., Gothard, K.M., and Markus, E.J. (1992). An Informationtheoretic Approach to Deciphering the Hippocampal Code (Morgan Kaufmann Publishers Inc.). Smith, M.A., and Kohn, A. (2008). Spatial and Temporal Scales of Neuronal Correlation in Primary Visual Cortex. Journal of Neuroscience 28, 12591-12603.

Speakman, A., and O'Keefe, J. (1990). Hippocampal Complex Spike Cells do not Change Their Place Fields if the Goal is Moved Within a Cue Controlled Environment. The European journal of neuroscience 2, 544-555.

Squire, L.R. (1986). Mechanisms of memory. Science (New York, NY) 232, 1612-1619.

Stein, B.E., Stanford, T.R., and Rowland, B.A. (2014). Development of multisensory integration from the perspective of the individual neuron. Nat Rev Neurosci 15, 520-535.

Taube, J.S. (1995). Head direction cells recorded in the anterior thalamic nuclei of freely moving rats. The Journal of Neuroscience: The Official Journal of the Society for Neuroscience 15, 7086.

Taube, J.S. (2007). The head direction signal: origins and sensory-motor integration. Annual Review of Neuroscience 30, 181-207.

Taube, J.S., Muller, R.U., and Ranck, J.B. (1990). Head-direction cells recorded from the postsubiculum in freely moving rats. II. Effects of environmental manipulations. Journal of Neuroscience 10, 436-447.

1318 Teyler, T.J., and DiScenna, P. (1985). The role of hippocampus in memory: a hypothesis. Neurosci Biobehav Rev 9, 377-389.

1321 Thompson, G.C., and Cortez, A.M. (1983). The inability of squirrel monkeys to localize sound after unilateral ablation of auditory cortex. Behavioural Brain Research 8, 211-216. 
Town, S.M., Brimijoin, W.O., and Bizley, J.K. (2017). Egocentric and allocentric representations in auditory cortex. PLOS Biology 15, e2001878.

Vélez-Fort, M., Bracey, E.F., Keshavarzi, S., Rousseau, C.V., Cossell, L., Lenzi, S.C., Strom, M., and Margrie, T.W. (2018). A Circuit for Integration of Head- and Visual-Motion Signals in Layer 6 of Mouse Primary Visual Cortex. Neuron 98, 179-191.e176. Wang, X., Liu, J., and Zhang, J. (2019). Chronic Unilateral Hearing Loss Disrupts Neural Tuning to Sound-Source Azimuth in the Rat Primary Auditory Cortex. Frontiers in Neuroscience 13. Whitlock, Jonathan R., Pfuhl, G., Dagslott, N., Moser, M.-B., and Moser, Edvard I. (2012). Functional Split between Parietal and Entorhinal Cortices in the Rat. Neuron 73, 789-802. egocentric and world-centered reference frames in the rat posterior parietal cortex. The Journal of Neuroscience: The Official Journal of the Society for Neuroscience 34, 5431-5446. Witter, M.P., Naber, P.A., van Haeften, T., Machielsen, W.C., Rombouts, S.A., Barkhof, F., Scheltens, P., and Lopes da Silva, F.H. (2000). Cortico-hippocampal communication by way of parallel parahippocampal-subicular pathways. Hippocampus 10, 398-410. Zaidel, A., DeAngelis, G.C., and Angelaki, D.E. (2017). Decoupled choice-driven and stimulusrelated activity in parietal neurons may be misrepresented by choice probabilities. Nature Communications 8, 715 . its implications for psychophysical performance. Nature 370, 140-143. 